\title{
Fluid structure of 1D spinful Fermi gases with long-range interactions
}

\author{
E Colella ${ }^{1}$, M L Chiofalo ${ }^{2}$, M Barsanti ${ }^{3}$, D Rossini $^{2}$, R Citro ${ }^{4}$ \\ ${ }^{1}$ Institut für Theoretische Physik, Universität Innsbruck, Technikerstrasse 21/3, \\ A-6020 Innsbruck, Austria \\ 2Dipartimento di Fisica "Enrico Fermi" and INFN, Università di Pisa, Largo Bruno \\ Pontecorvo 3, I-56127 Pisa, Italy \\ ${ }^{3}$ Dipartimento di Ingegneria Civile e Industriale dell'Università di Pisa and INFN, \\ Università di Pisa, Largo Lucio Lazzarino, I-56124 Pisa, Italy \\ ${ }^{4}$ Dipartimento di Fisica "E.R. Caianiello", Università degli Studi di Salerno, Via \\ Giovanni Paolo II, 132 I-84084 Fisciano (Sa), Italy and INFN Sezione di Napoli, \\ Gruppo collegato di Salerno, Italy
}

\begin{abstract}
We discuss the fluid structure in the quantum phases of a $1 \mathrm{D}$ spinful Fermi gas of atoms interacting via an infinitely long-range coupling, as it may result from a photon-mediated two-body coupling in optical cavities. The system reveals a rich physics, where spin/charge-density wave and superfluid-like order compete with each other. Following our previous work based on a combined mean-field, exact diagonalization and bosonization analysis, we provide the phase diagram of the system and discuss the structure of the fluid, addressing the main features in momentum space of the order parameters, momentum distribution and two-body correlations. We enlighten that the nesting of the Fermi surface in 1D ultimately drives the formation of periodic structures commensurate with the cavity-induced mean-field potential.
\end{abstract}

Submitted to: J. Phys. B: At. Mol. Opt. Phys. 


\section{Introduction}

Quantum-degenerate ultracold gases represent an optimal platform for the simulation of problems which are interesting for different areas of physics, ranging from the fundamental to the many-body realm. Due to the possibility of careful handling of temperature, statistics, dimensionality, strength and range of the atomic interactions, they allow to reach extreme quantum conditions [1-3]. Among current, and most relevant, many-body problems, the relation between spin-density wave (SDW), charge-density wave (CDW), and superfluid (SF) order in FeAs-based superconductors [4-6] is recently attracting a great deal of experimental and theoretical interest. Indeed, synthetic engineering of systems which display similar behaviour will lead to a better understanding of the important microscopic mechanisms beyond high-temperature superconductivity $[7,8]$, characterized by non-Fermi liquid behavior in the non-superconducting phase. On general grounds, the interplay between spin and density excitations is central to the physics of quantum fluids confined in reduced dimensions. It is especially interesting to disentangle the underlying physics in the one-dimensional (1D) case. Here, the spin-charge separation leads to the concept of the Luttinger-liquid quantum fluid, which represents the ideal framework where to search for novel quantum phase diagrams in the presence of long-range interactions, doping, incommensurations, and magnetic-like effects [9].

Among all the possible quantum gases platforms, optical cavities stand as particularly versatile realizations where to explore this rich phenomenology. In such a kind of setups, the atom-photon scattering probability is enhanced with respect to free space by the many round-trips of the photons in the cavity [10]. Cavity photons can indeed mediate interactions between atoms, originally characterized by an infinite range driven by characteristic mode function of the cavity field, i.e. the stationary or running waves for a linear or ring cavity, respectively [11]. The effective interaction may in principle affect both the internal and motional degrees of freedom, depending on the particular pumping configuration and original atomic structure [12-16]. In addition, the optical cavity parameters can also be tailored to tune the effective interactions in strength and sign [17], or even to tune short-range interactions in multi-mode cavities [18].

In this framework, a considerable research activity has been devoted to the effects of cavity photons on either spin or momentum degrees of freedom. With these tools at hand, a number of investigations have been conducted, including the study of the density properties of superradiant systems in thermal atoms [19, 20], BECs [21-23], and Fermi gases [24-26], topological phase transitions [14, 13], artificial gauge fields [27-29] and supersolidity [30,31]. The more recent experimental realization of magnetic phases in spinor BECs [32, 18, 33] motivates theoretical studies on superradiant systems with spin dynamics, spin-orbit coupling [34, 35] and the crossover from a Bardeen-Cooper-Schrieffer (BCS) superfluidity of Cooper pairs to a BEC of composite bosonic pairs mediated by cavity photons [36].

Actual implementations of many-body physics in optical cavities require a careful 
handling of the unavoidable dissipation processes and non-equilibrium dynamics. Even with this in mind, the tools offered by cold atoms in optical resonators represent a reference framework to investigate the nature of the quantum phases of 1D Fermi systems with tunable interactions, in terms of the combined action of spin and density excitations.

Along these lines, using combined mean-field (MF), exact diagonalization (ED) and bosonization methods, in reference [37] we explored the quantum phases of a 1D spinful Fermi fluid in the presence of a photon-mediated two-body effective interaction of strength $g$ among the atoms, obtained after adiabatic elimination of the cavity photons within a single-mode approximation, so that the effective interaction turns out to be infinitely long ranged. We found that, as expected in 1D, cavity photons drive strong correlations independently of the interaction strength $|g|$. On the other hand, the sign of $g$, which can in principle be modified turning from red to blue the cavity detuning from the pump frequency, determines whether an instability occurs in the particle-hole $(g<0)$ or particle-particle $(g>0)$ channel. The resulting phase diagram is composed by a Spin-Density-Wave (SDW)-like ordering directly induced by the interaction of the two-level atoms with cavity photons, as well as Atomic Density Wave (ADW)-like and superfluid (SF) ordering indirectly induced via spin fluctuations.

The study performed in reference [37] deliberately uses a combination of methods, since a direct simulation by, e.g. Density-Matrix Renormalization Group-like methods in the presence of long-range interactions is not straightforward. First, a MF analysis was conducted, in order to get a qualitative guide to the underlying physics. In fact, it is generally found [9] that in the presence of (infinitely) long-range interactions as in our case, the MF approximation in $1 \mathrm{D}$ is able to capture the qualitative picture of the system properties. The conclusions drawn from the MF analysis were then checked against an ED method, suited to explore the correlation functions and the momentum distribution. On the other hand, ED does not yield immediate access to the SF order parameter. Therefore, we have also resorted to bosonization methods to infer the occurrence of SF ordering from the divergence of the appropriate susceptibilities, as anticipated in the MF analysis. The bosonization analysis has also provided a cross-checking with the ED results on the appearance of SDW and ADW orderings. Overall, our combined methods lead to the conclusion that, as expected for 1D systems in the presence of (infinitely) long-range interactions, the MF approximation is able to provide a picture which qualitatively agrees with the main conclusions drawn with ED and bosonization methods. Here, we provide a detailed account of the spectral properties of the fluid, which helps to clarify the nature of the different phases. We discuss detailed results obtained within the MF analysis, remarking the comparison with the results from ED and bosonization methods whenever needed.

The paper is organized as follows. In sections 2 and 3 we summarize the essentials of our model and the methods used to obtain the phase diagram, which is discussed in section 4. This material is mainly summarized from reference [37]. In section 5, we illustrate the fluid structure, as it emerges from the analysis of the gaps and pair- 
correlation functions across the phase diagram. Concluding remarks and perspectives are presented in section 6 .

\section{Model}

We consider the following many-body Hamiltonian for 1D fermions with mass $m$, placed in two spin levels $\uparrow, \downarrow$ :

$$
\hat{H}=\sum_{\sigma=\uparrow, \downarrow} \int d x \hat{\Psi}_{\sigma}^{\dagger}(x)\left[-\frac{\hbar^{2} \nabla^{2}}{2 m}\right] \hat{\Psi}_{\sigma}(x)+\hbar g \int d x d x^{\prime} V\left(x, x^{\prime}\right) \hat{\Psi}_{\downarrow}^{\dagger}(x) \hat{\Psi}_{\uparrow}(x) \hat{\Psi}_{\uparrow}^{\dagger}\left(x^{\prime}\right) \hat{\Psi}_{\downarrow}\left(x^{\prime}\right),
$$

in terms of destruction (creation) fermionic field operators $\hat{\Psi}_{\sigma}^{(\dagger)}(x)$. While referring to Appendix A for details, the above effective Hamiltonian (1) can be obtained by transversally pumping $N$ three-level atoms in an optical cavity. By eliminating the far-detuned excited level and the cavity fields in the bad cavity regime, an effective Hamiltonian for interacting fermions can be realized. The fermions in the two remaining ground states can be treated as a spin- $1 / 2$ system interacting via the infinitely long-range potential

$$
V\left(x, x^{\prime}\right)=\cos \left(k_{L} x\right) \cos \left(k_{L} x^{\prime}\right),
$$

due to the cavity electromagnetic field with wavevector $k_{L}$. Here, we assumed that the classical energy shift induced by the two-photon transition can be compensated by an external light shift. The coupling

$$
g=g_{\mathrm{eff}}^{2} \frac{\Delta_{c}}{\kappa^{2} / 4+\Delta_{c}^{2}}
$$

can be, in principle, varied with the cavity detuning $\Delta_{c}=\omega_{p}-\omega_{c}$, where $\omega_{c}$ is the frequency of the cavity mode and $\omega_{p}$ the one of the transversal pump laser. Here, $\kappa$ is the cavity-loss parameter and the effective two-photon Rabi coupling $\hbar g_{\text {eff }} \equiv \hbar g_{0} \Omega / \Delta_{e}$ is given in terms of the original cavity-mode strength $\hbar g_{0}$, and the Rabi frequency $\Omega$ of the classical field transversely pumping the atomic transition. Finally, $\Delta_{e}=\omega_{p}-\omega_{e}$, is the detuning of the upper (eliminated) level from the pump frequency.

A glance at all the different possible pairings in the interaction term of Hamiltonian (1), suggests that the relevant processes to expect can be driven by spin and density fluctuations, and by superfluid pairing. We have determined the ground-state properties of equation (1) while varying $g$, by means of a combination of standard MF treatment, $\mathrm{ED}$, and bosonization analysis. 


\section{Methods}

\subsection{Mean-field approach}

Let us start to discuss the MF method, temporarily leaving the density fluctuations on a side. The SF gap is defined as:

$$
\Delta(k)=-g \sum_{k^{\prime}} V_{k, k^{\prime}}\left\langle\hat{c}_{-k^{\prime}, \downarrow} \hat{c}_{k^{\prime}, \uparrow}\right\rangle
$$

where $V_{k, k^{\prime}}=\delta\left(k^{\prime}-k+k_{L}\right)+\delta\left(k^{\prime}-k-k_{L}\right)$ is the Fourier transformed microscopic interaction potential in equation $(1)$, and $\hat{c}_{k, \sigma}^{(\dagger)}$ the destruction (creation) operators in $k$ space. Notice that hereafter we adopt the notation $\langle\cdot\rangle$ to indicate ground-state expectation values of any operator. Likewise, we introduce the SDW gap:

$$
S(Q)=-g \sum_{k}\left\langle\hat{c}_{k, \downarrow}^{\dagger} \hat{c}_{k+Q, \uparrow}\right\rangle
$$

which is related to spin waves propagating at the wave-vector $Q= \pm k_{L}$ of the cavity field.

We derive the coupled equations of motion in imaginary time for the four Green's functions, $-\left\langle\vec{T}_{\tau}\left[\hat{c}_{p, \sigma}(\tau) \hat{c}_{p, \sigma}^{\dagger}\left(\tau^{\prime}\right)\right]\right\rangle,-\left\langle\vec{T}_{\tau}\left[\hat{c}_{p, \uparrow}(\tau) \hat{c}_{-p, \downarrow}\left(\tau^{\prime}\right)\right]\right\rangle,-\left\langle\vec{T}_{\tau}\left[\hat{c}_{p, \uparrow}(\tau) \hat{c}_{p+Q, \downarrow}^{\dagger}\left(\tau^{\prime}\right)\right]\right\rangle$, and $-\left\langle\vec{T}_{\tau}\left[\hat{c}_{k, \uparrow}(\tau) \hat{c}_{-k-Q, \uparrow}\left(\tau^{\prime}\right)\right]\right\rangle$, from which the self-consistent equations for the number of particles $N$, and the two superfluid $\Delta(k)$ and spin $S(Q)$ gaps can be obtained.

Though the resulting equations are still quite involved, the formulation can be greatly simplified after considering one characteristic peculiarity of $1 \mathrm{D}$ systems, that is the nesting property $\varepsilon\left(k+2 k_{F}\right)=-\varepsilon(k)$ which in 1D characterizes the single-particle excitation energy $\varepsilon_{k} \equiv \hbar^{2} k^{2} /(2 m)-\mu$ referred to the chemical potential $\mu$, with $k_{F}$ being the Fermi momentum. In fact, in $1 \mathrm{D}$ the Fermi surface is given by the two points $\pm k_{F}$, which are entirely nested, i.e. connected by a single wave-vector, here given by $Q=2 k_{F}$. As is well-known, this condition is responsible for diverging susceptibilities in the particle-hole and, possibly, particle-particle channel, in analogy with Cooper instability [9]. Then, we get additional physical insight by considering the two gap equations obtained for $\Delta_{\mathrm{SF}} \equiv \sum_{k} \Delta(k)$ after setting $\Delta_{\mathrm{SDW}}^{2} \equiv \sum_{Q= \pm 2 k_{F}}[S(Q)]^{2}=0$, and vice versa. When setting the spin-gap parameter to zero, one has [37]:

$$
\Delta(k)=g \sum_{k^{\prime}} V_{k k^{\prime}} \frac{\Delta\left(k^{\prime}\right)}{2 E_{\mathrm{SF}}\left(k^{\prime}\right)} \tanh \left(\frac{\beta E_{\mathrm{SF}}\left(k^{\prime}\right)}{2}\right)
$$

with the excitation energy

$$
E_{S F}(k) \equiv \sqrt{\epsilon_{k}^{2}+\Delta(k)^{2}}
$$

and the number equation

$$
N=2 \sum_{k}\left[\frac{1}{2}-\frac{\epsilon_{k}}{2 E_{\mathrm{SF}}(k)} \tanh \left(\frac{\beta E_{\mathrm{SF}}(k)}{2}\right)\right] \equiv 2 \sum_{l} n(k) .
$$


On the other hand, when setting the superfluid gap to zero, one obtains:

$$
S(Q)=-g \sum_{k} \frac{S(Q)}{2 E_{\mathrm{SDW}}(k)} \tanh \left(\frac{\beta E_{\mathrm{SDW}}(k)}{2}\right),
$$

with the excitation energy

$$
E_{\mathrm{SDW}}(k) \equiv \sqrt{\epsilon_{k}^{2}+\Delta_{\mathrm{SDW}}^{2}}
$$

$\Delta_{\mathrm{SDW}}^{2} \equiv \sum_{Q= \pm 2 k_{F}}[S(Q)]^{2}$, and the number equation

$$
N=2 \sum_{k}\left[\frac{1}{2}-\frac{\epsilon_{k}}{2 E_{\mathrm{SDW}}(k)} \tanh \left(\frac{\beta E_{\mathrm{SDW}}(k)}{2}\right)\right] .
$$

The sign consistency between both members in the SF equation (6) is only fulfilled for $g>0$. For analogous reasons, the SDW equation (9) admits solutions only for $g<0$. If this property persists in the coupled equations, where both gaps are set to non-zero values, this would imply that SF and SDW ordering cannot coexist. This is indeed the case, as we have numerically checked by solving the full set of coupled equations [37]. In order to be self-contained, we have reported in Appendix B the details of the derivation.

Let us finally note that, although the MF equations above are derived at finite temperature, we are interested in the solution at $T=0$ where the effect of fluctuations can be neglected.

\subsection{Exact diagonalization}

Even though the long-range nature of the interactions usually preludes to qualitatively reliable MF solutions, in order to check the MF results and also to include atomic-density wave (ADW) fluctuations into play, we have employed an ED analysis in momentum space. We diagonalize the Hamiltonian for a system of length $\ell$ at fixed density $n k_{F}=2 / \pi$. We adopted a Lanczos-based algorithm, enabling the diagonalization of up to $\sim\left(10^{7} \times 10^{7}\right)$ matrices on a laptop computer. These sizes allow us to perform the computation for systems with $N=4$ and $L=21, N=8$ and $L=19, N=12$ and $L=15$. Here, $L$ denotes the number of discretized $k$-points adopted in the simulation. We employ a constant mesh $\Delta k=2 \pi / \ell$ for the grid in momentum space.

Besides the ground-state energy and the momentum distribution function, we have computed density-density and spin-spin structure factors, i.e. the Fourier transform of the correlation functions $[9]\left\langle\hat{O}_{\rho}(x) \hat{O}_{\rho}(0)\right\rangle$ and

$$
\left\langle\hat{O}_{\sigma}(x) \hat{O}_{\sigma}(0)\right\rangle \equiv \sum_{\alpha=x, y}\left\langle\hat{O}_{s}^{\alpha}(x) \hat{O}_{s}^{\alpha}(0)\right\rangle
$$

with the definitions

$$
\begin{aligned}
& \hat{O}_{\rho}(x) \equiv \sum_{\sigma} \hat{\Psi}_{\sigma}^{\dagger}(x) \hat{\Psi}_{\sigma}(x), \\
& \hat{O}_{s}^{x}(x) \equiv \hat{\Psi}_{\uparrow}^{\dagger}(x) \hat{\Psi}_{\downarrow}(x)+\hat{\Psi}_{\downarrow}^{\dagger}(x) \hat{\Psi}_{\uparrow}(x), \\
& \hat{O}_{s}^{y}(x) \equiv-i\left[\hat{\Psi}_{\uparrow}^{\dagger}(x) \hat{\Psi}_{\downarrow}(x)-\hat{\Psi}_{\downarrow}^{\dagger}(x) \hat{\Psi}_{\uparrow}(x)\right] .
\end{aligned}
$$




\subsection{Bosonization method}

Unfortunately, the applicability of ED is restricted to small $N$ and $L$ values, and to the microcanonical ensemble, where the phase-locked SF ground state is not retrievable. In order to explore possible SF phases beyond $\mathrm{MF}$, we applied a bosonization technique. We introduced the bosonized fields for the fermionic operators [9], $\hat{\Psi}_{\sigma}(x)=e^{-i k_{F} x} \hat{\Psi}_{R \sigma}(x)+e^{i k_{F} x} \hat{\Psi}_{L \sigma}$, where $\hat{\Psi}_{r \sigma}(x)$ are the right/left movers $(r=R, L$ or idem $r= \pm$ below) with spin $\sigma=\uparrow, \downarrow$ :

$$
\hat{\Psi}_{r \sigma}(x)=\frac{1}{\sqrt{2 \pi \alpha}} e^{\frac{i}{\sqrt{2}}\left[\hat{\theta}_{\rho}(x)-r \hat{\phi}_{\rho}(x)+\sigma\left(\hat{\theta}_{\sigma}(x)-r \hat{\phi}_{\sigma}(x)\right)\right]},
$$

while $\alpha$ is the cutoff of the theory, in particular $\alpha \sim a, a$ being the lattice spacing. The bosonic fields $\left(\hat{\phi}_{\rho}, \hat{\theta}_{\rho}\right)$ and $\left(\hat{\phi}_{\sigma}, \hat{\theta}_{\sigma}\right)$ are linked to the charge and spin channel, respectively. Retaining only the slowly oscillating terms of the bosonized expansion, the bosonized Hamiltonian for $k_{L}=2 k_{F}$, becomes:

$$
\begin{aligned}
\hat{H}_{B} & \sim \sum_{l=\rho, \sigma} \frac{1}{2 \pi} \int d x\left[u_{l} K_{l}\left(\pi \hat{\Pi}_{l}(x)\right)^{2}+\frac{u_{l}}{K_{l}}\left(\nabla \hat{\phi}_{l}(x)\right)^{2}\right] \\
& +\frac{g}{4 \pi^{2} \alpha^{2}} \iint d x d x^{\prime} e^{-i \sqrt{2}\left(\hat{\theta}_{\sigma}(x)-\hat{\theta}_{\sigma}\left(x^{\prime}\right)\right)} \cos \left[\sqrt{2}\left(\hat{\phi}_{\rho}(x)-\hat{\phi}_{\rho}\left(x^{\prime}\right)\right)\right],
\end{aligned}
$$

with $\hat{\Pi}_{l}=\nabla \hat{\theta}_{l}$, and $\left(u_{l}, K_{l}\right)$ being the Luttinger parameters for the corresponding two channels. Here, $u_{l}=v_{F}$ is the Fermi velocity and $K_{l}=1$ for non-interacting fermions. We notice that the intrinsically non-local nature of the interaction Hamiltonian, due to the long-range nature of photon-mediated interaction, emerges in the coupling of the fields from the spin $\hat{\theta}_{\sigma}$ and charge $\hat{\phi}_{\rho}$ sectors at different positions. Let us also note that the longer-range interaction does not invalidate the Luttinger liquid description as long as $V(x)$ has a finite Fourier transform for $q \rightarrow 0$. In fact, since we are interested in the long-range part of the interaction, one can keep in the density only the $q \sim 0$ part and if $V(x)$ has a finite Fourier transform for $q \rightarrow 0$, then the Luttinger parameters tend to a constant at very small $q$. Thus the asymptotic form of the correlation functions is exactly identical to the ones of a Luttinger liquid.

In order to infer the phase diagram of the model, we look at the analytic continuation of the Fourier transforms of the following correlation functions:

$$
\chi_{a}\left(k, \omega_{n}\right) \equiv \int d r \int_{0}^{\beta} d \tau e^{\left(-i k r+i \omega_{n} \tau\right)}\left\langle\hat{O}_{a}^{\dagger}(r) \hat{O}_{a}(0)\right\rangle
$$

characterizing $\mathrm{SDW}_{x-y}, \mathrm{ADW}$ and singlet (SS)/triplet (TS) superfluidity [9]:

$$
\begin{array}{ll}
\left\langle\hat{O}_{\mathrm{SDW}}^{x-y \dagger}(r) \hat{O}_{\mathrm{SDW}}^{x-y}(0)\right\rangle, & \left\langle\hat{O}_{\mathrm{ADW}}^{\dagger}(r) \hat{O}_{\mathrm{ADW}}(0)\right\rangle, \\
\left\langle\hat{O}_{\mathrm{SS}}^{\dagger}(r) \hat{O}_{\mathrm{SS}}(0)\right\rangle, & \left\langle\hat{O}_{\mathrm{TS}}^{\dagger}(r) \hat{O}_{\mathrm{TS}}(0)\right\rangle,
\end{array}
$$


with the operators defined as

$$
\begin{aligned}
& \hat{O}_{\mathrm{SDW}}^{x}=\hat{\Psi}_{R \uparrow}^{\dagger} \hat{\Psi}_{L \downarrow}+\hat{\Psi}_{R \downarrow}^{\dagger} \hat{\Psi}_{L \uparrow}, \\
& \hat{O}_{\mathrm{SDW}}^{y}=-i\left(\hat{\Psi}_{R \uparrow}^{\dagger} \hat{\Psi}_{L \downarrow}-\hat{\Psi}_{R \downarrow}^{\dagger} \hat{\Psi}_{L \uparrow}\right), \\
& \hat{O}_{\mathrm{ADW}}=\hat{\Psi}_{R \uparrow}^{\dagger} \hat{\Psi}_{L \uparrow}+\hat{\Psi}_{R \downarrow}^{\dagger} \hat{\Psi}_{L \downarrow}, \\
& \hat{O}_{\mathrm{SS}}=\hat{\Psi}_{R \uparrow}^{\dagger} \hat{\Psi}_{L \downarrow}^{\dagger}+\hat{\Psi}_{L \uparrow}^{\dagger} \hat{\Psi}_{R \downarrow}^{\dagger}, \\
& \hat{O}_{\mathrm{TS}}=\hat{\Psi}_{R \uparrow}^{\dagger} \hat{\Psi}_{L \downarrow}^{\dagger}-\hat{\Psi}_{L \uparrow}^{\dagger} \hat{\Psi}_{R \downarrow}^{\dagger},
\end{aligned}
$$

For a qualitative comparison with the ED and MF outcomes, it is sufficient to look at the behaviour of the correlation functions (17) as described in terms of the Luttinger parameters. Since we know the long wavelength behavior of the correlation functions, for a qualitative comparison it is sufficient to infer the behaviour of the Luttinger parameters $K_{\rho}$ and $K_{\sigma}$, while varying the interaction strength. Indeed, the most diverging correlation functions reveal whether the system is unstable towards an Atomic-Density Wave (ADW), a Spin-Density Wave (SDW) or a Superfluid phase, either with singlet (SS) or triplet (TS) order parameter. To start with, we approach the problem within a mean-field scheme and decouple the interaction term in (15) considering separately the cases where either one of the fields $\hat{\theta}_{\sigma}$ or $\hat{\phi}_{\rho}$ becomes massive. When the term $\Delta=\left\langle e^{i \hat{\theta}_{\sigma}}\right\rangle$ acquires an average different from zero, the system will open a gap in the charge sector when the operator $\cos \left(\sqrt{2} \hat{\phi}_{\rho}\right)$ becomes relevant, i.e. $K_{\rho}<3$ and this behavior is independent on the sign of the interaction $g$. On the other hand, when $\hat{\phi}_{\rho}$ becomes massive and is trapped in one of the minima of the cosine, or equivalently, $\Gamma=\left\langle\cos \left(\sqrt{2} \hat{\phi}_{\rho}\right)\right\rangle$ is nonzero, a gap in the spin sector is opened when the operator $e^{i \hat{\theta}_{\sigma}}$ becomes relevant. This happens when $K_{\sigma}>1 / 3$ for $g>0$. Thus one expects the instabilities of the system be different depending on the sign of the interaction $g$. A more refined analysis is given by a Renormalization Group ( $R G$ ) study which gives the renormalized values of the Luttinger parameters $K_{\rho}, K_{\sigma}$, allowing to evaluate the regions where the susceptibilities diverge as a function of Luttinger parameters. Appendix $\mathrm{C}$ details the derivation of the RG equations. Notwithstanding its qualitative character, the bosonization approach is consistent with and supports the outcomes of the MF and ED studies addressing the channel where the instability preferably manifests.

\section{Phase Diagram}

We are now in the position to review the basic results for the phase diagram and the fluid structure of the model. Let us remind that, while this has been already discussed in reference [37], for the sake of clarity in our presentation, it is however helpful to provide the reader with an excerpt of the main findings, as summarized in Figure 1. Here, the two different order parameters $\Delta_{\mathrm{SDW}}$ and $\Delta_{\mathrm{SF}}$ are displayed, at the MF level, as a function of the coupling strength $g$ in $E_{F}$ units. We first notice that, based on these results, $\Delta_{\mathrm{SDW}}$ ordering in the particle-hole channel develops for negative $g$ values, while $\Delta_{\mathrm{SF}}$ ordering in the particle-particle channel shows up at $g>0$. In close analogy to 
references [16, 38-40], on the side $g<0$ an antiferromagnetic structure is found to be stabilized. In this regime, the system exhibits superradiance and the fluctuations of the cavity field can be neglected [39]. In contrast, in the regime $g>0$, atoms are trapped in a dark state. While we found that, at the MF level, the induced interaction can lead in this case to superconducting ordering, in this regime quantum fluctuations can play a fundamental role, leading to density waves [39] or ferromagnetic and incommensurate magnetic phases [38].

In order to address some of the physics beyond MF, we carried out a bosonization analysis, which supports the MF findings, and provides information on the ADW phase which was left on a side in the MF calculation. In fact, the RG analysis on the susceptibilities (17) establishes that, for $g<0, K_{\rho}$ and $K_{\sigma}$ get values leading to divergent behavior $\mathrm{SDW}_{x-y} \sim \omega^{K_{\rho}+K_{\sigma}^{-1}-2}$ of the $\mathrm{SDW}_{x-y}$ susceptibility for $k=2 k_{F}$, while all the others turn out to be well behaved. For $g>0$ the singlet superfluid correlation function gets the most diverging behavior $\chi_{\mathrm{SS}} \sim \omega^{K_{\rho}^{-1}+K_{\sigma}-2}$. This suggests that the dominant instability may be in the form of singlet superfluidity. No sign of ADW is found in this low-order bosonization analysis, signaling the necessity of expanding the bosonized fields beyond the lowest order and include the effects of the fast modes, up to $k_{L}=2 k_{F}$ and of doing higher-order renormalization group. As it will be shown in the following, an indication of $\mathrm{ADW}$ can be traced in the emergence of peaks at $k_{L}=2 k_{F}$ and $2 k_{L}=4 k_{F}$, in the density-density correlation function calculated via ED. This is especially relevant, since it was shown that quantum measurements in 1D lattices can lead to excitations far from the Fermi surface [41]. Overall, the MF results and the bosonization analysis seem to support the idea that spin-density fluctuations and superfluidity do not coexist. In addition, ED confirms the magnetic phases arising from SDW for $g<0$ and hints the presence of ADW at $g>0$, which was indeed also found in [39].

Second, we notice that the size of the order parameters increases with increasing $|g|>1$ values, but in a very asymmetric manner. We believe that this behavior can be traced back to the physics of the original system, where the spin-flip processes are a direct consequence of the emission or absorption of a cavity photon. In many-body physics, this is what one would call a spin-density fluctuation, which is indeed the original mechanism inducing, only at a second stage, SF or (even higher in order) ADW fluctuations. It is interesting to notice that trace of this asymmetric behavior can be found also in the dependence of the ground-state energy on the interaction strength, as computed from ED methods [37].

Additional insight can be inferred from the behavior of the chemical potential $\mu$ and fraction of particles in the lowest energy state,

$$
N_{0} \equiv \sum_{k, Q}\left|\left\langle\hat{c}_{k+Q \downarrow}^{\dagger} \hat{c}_{k \uparrow}\right\rangle\right|^{2}
$$

in the SDW region with $g<0$, in analogy with the usual BCS definition $N_{0}=$ $\sum_{k}\left|\left\langle\hat{c}_{-k \downarrow} \hat{c}_{k \uparrow}\right\rangle\right|^{2}$, used in the SF region with $g>0$. From the insets of figure 1, we see that the chemical potential is at the Fermi energy level $E_{F}$ in the SF region with 
$g>0$, decreasing in the SDW region with $g<0$ up to get negative values around $|g| \simeq 8 E_{F}$. Correspondingly, $N_{0}$ increases while $|g|>1$, but in a similar very asymmetric manner as the order parameters, i.e. to significantly larger values on the SDW than on the SF side.

\section{Structure of the fluid}

We now proceed to characterize the nature of the ground states emerging in the phase diagram of figure 1, by analyzing the structure of the gaps, the excitation spectra, the momentum distributions, and the pair-correlation functions, as they result from the MF solution. Whenever useful, we provide additional information which can be extracted from ED and bosonization analysis.

\subsection{Order parameters}

Let us start with the order parameters. In the SDW region $(g<0)$, the MF result for the gap function $S(Q)$ is a delta function of the cavity wave-vector $Q= \pm k_{L}= \pm 2 k_{F}$. In fact, the order parameter $S(Q)$ is associated to particle-hole excitations propagating at the momentum of the cavity photons $\pm k_{L}$. For weak interactions, these excitations are created at the Fermi surface. However, as the interaction strength is increased, more particles below the Fermi surface are scattered to higher momentum states. This can be

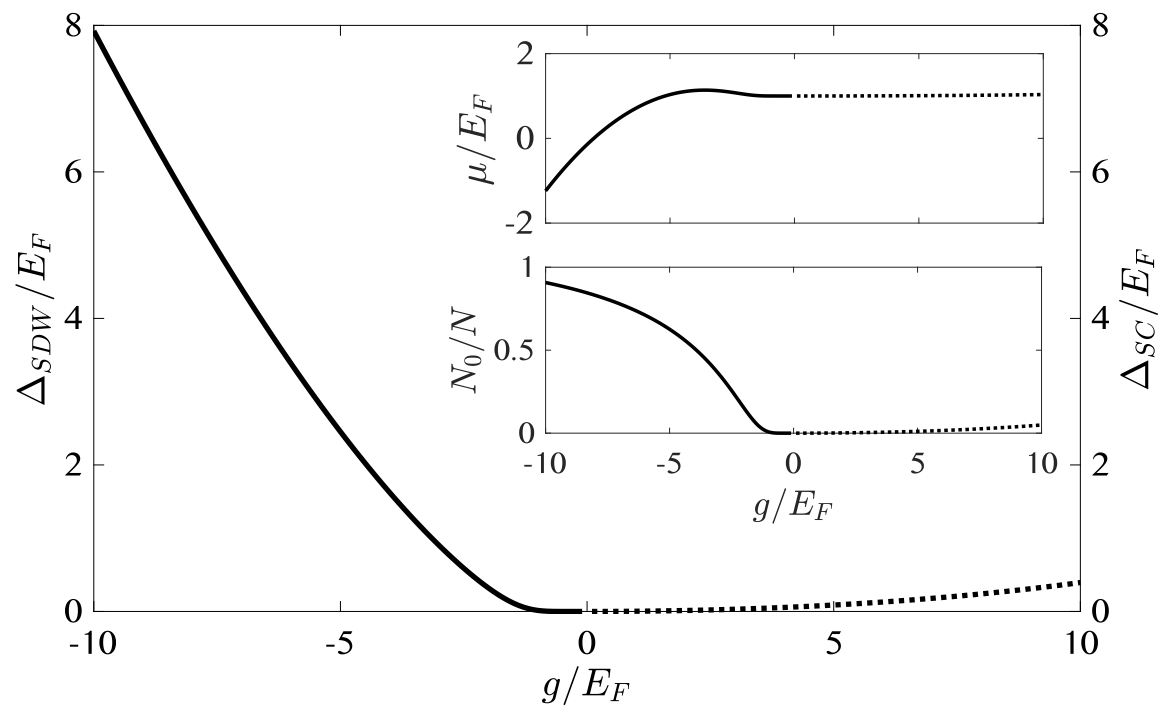

Figure 1. Mean-field phase diagram of the model as a function of the coupling strength $g$, from reference [37]. The MF order parameters $\Delta_{\mathrm{SDW}}$ and $\Delta_{\mathrm{SF}}$ are plotted against $g / E_{F}$. Insets: chemical potential $\mu / E_{F}$ (top) and fraction $N_{0} / N$ of particles in the lowest energy state, as functions of $g / E_{F}$. Adapted from [37]. 
examined in the momentum dependent order parameter $S_{Q}(k)$, which is given by the normalized pairing function

$$
S_{Q}(k)=\frac{\left\langle\hat{c}_{k+Q \downarrow}^{\dagger} \hat{c}_{k \uparrow}\right\rangle}{S(Q)}=\frac{1}{2 E_{\mathrm{SDW}}(k)} \tanh \left\{\frac{\beta E_{\mathrm{SDW}}(k)}{2}\right\} .
$$

As it is shown in figure 2, for weak interactions $S_{Q}(k)$ is seen to develop two peaks at $\pm k_{F}$, signaling the occurrence of spin ordering. The peaks broaden up to merging, when the chemical potential becomes negative at $g \sim-8 E_{F}$.

Coming to the SF region $(g>0)$, we first notice that in standard BCS theory the superfluid order parameter represents the energy required to break a Cooper pair. The symmetries of the interatomic potential directly affect those of the gap function. In our case, the microscopic interaction resulting from the coupling with photons is characterized by a sinusoidal modulation in real space, and therefore in momentum space by a sum of peaks at the momentum of the exchanged photon. At variance with standard treatments of the BCS equations where the gap is constant, here we expect a $k$ dependence with the periodicity driven by the cavity photon momentum.

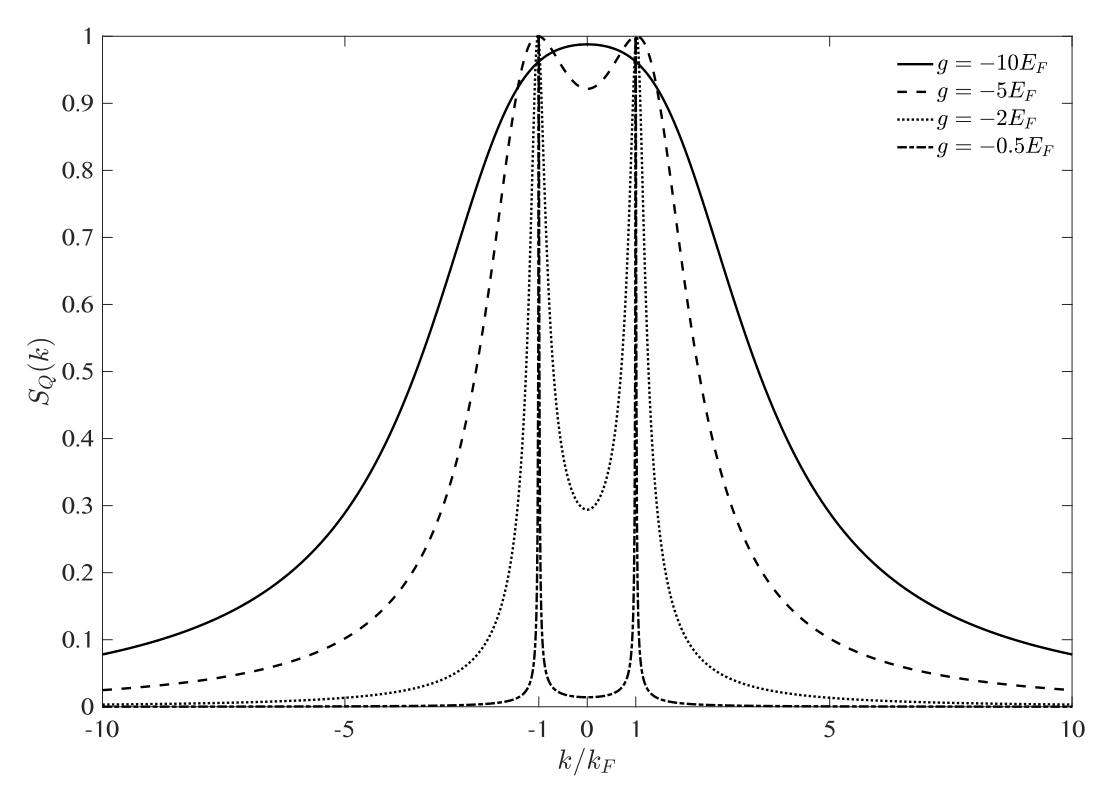

Figure 2. Pairing function $S_{Q}(k)$, as defined in the main text, as a function of $k / k_{F}$. The values of the coupling strength, $g<0$, are indicated in the legend

Figure 3 displays the behavior of the superfluid order parameter $\Delta(k)$ at different values of the coupling strength $g>0$, as mediated by the interaction with the cavity photons. We recall that $\Delta(k)=0$ at all $k$, in the $g<0$ side. Notice first that the superfluid gap is characterized by peaks separated by $Q=2 k_{F}$ and at odd multiples of the $k_{F}$ Fermi momentum, since the first peaks are located at $k= \pm k_{F}$. These repetitions can 


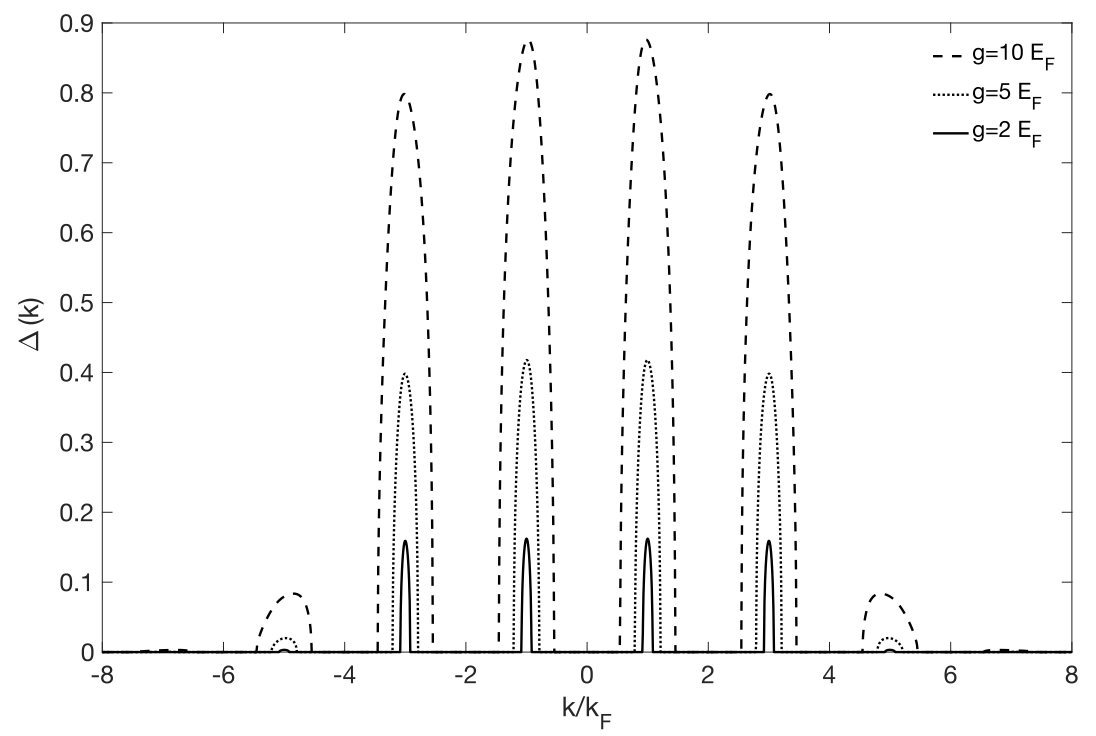

Figure 3. Superfluid order parameter $\Delta(k)$ as a function of $k / k_{F}$, for different values of the coupling strength $g>0$, as indicated in the legend.

be interpreted as the usual peaks arising from excitations near the Fermi level, shifted by the momentum recoil due to the exchanged photon $Q=2 k_{F}$. Their width increases while the interaction $g$ becomes stronger. In other words the recoil energy, which represents the difference of kinetic energy of the atom after a scattering with a photon, introduces an additional energy scale in our system. In particular, this is responsible for the number of visible peaks in the $k$-structure of the order parameter, while the interaction strength drives their width and height. The stability of the solution has been checked while trying different initial guesses of regular shape, starting the simulation from large values of $g$ going towards small values of $g$, and vice versa. Notice also that the overall plateau envelope characterizes $\Delta(k)$, which crashes down (exponentially, from our fits) already after the second peak.

\subsection{Excitation spectrum}

The same physics is reflected in the excitation spectra calculated within the MF approach, as shown in figure 4. The top panel displays the behavior in the SDW region $(g<0)$, where a gap is seen to open up at the Fermi level, which then progressively shifts toward $k=0$. Together with the fact that the chemical potential gets negative around $g \sim-8 E_{F}$, and that the condensate fraction changes its convexity, this supports the conclusion that the system is evolving towards a bosonic-like condensation regime. In fact, when the chemical potential turns negative, the kinetic energy cannot compensate it any longer at any value of $k$ and the chemical potential acts as a constant shift. On the other hand, the contribution of the spin gap to the excitation energy extends over all 
the Fermi surface: therefore, it is not surprising that the minimum of the energy shifts to zero when the kinetic contribution is at its minimum value.
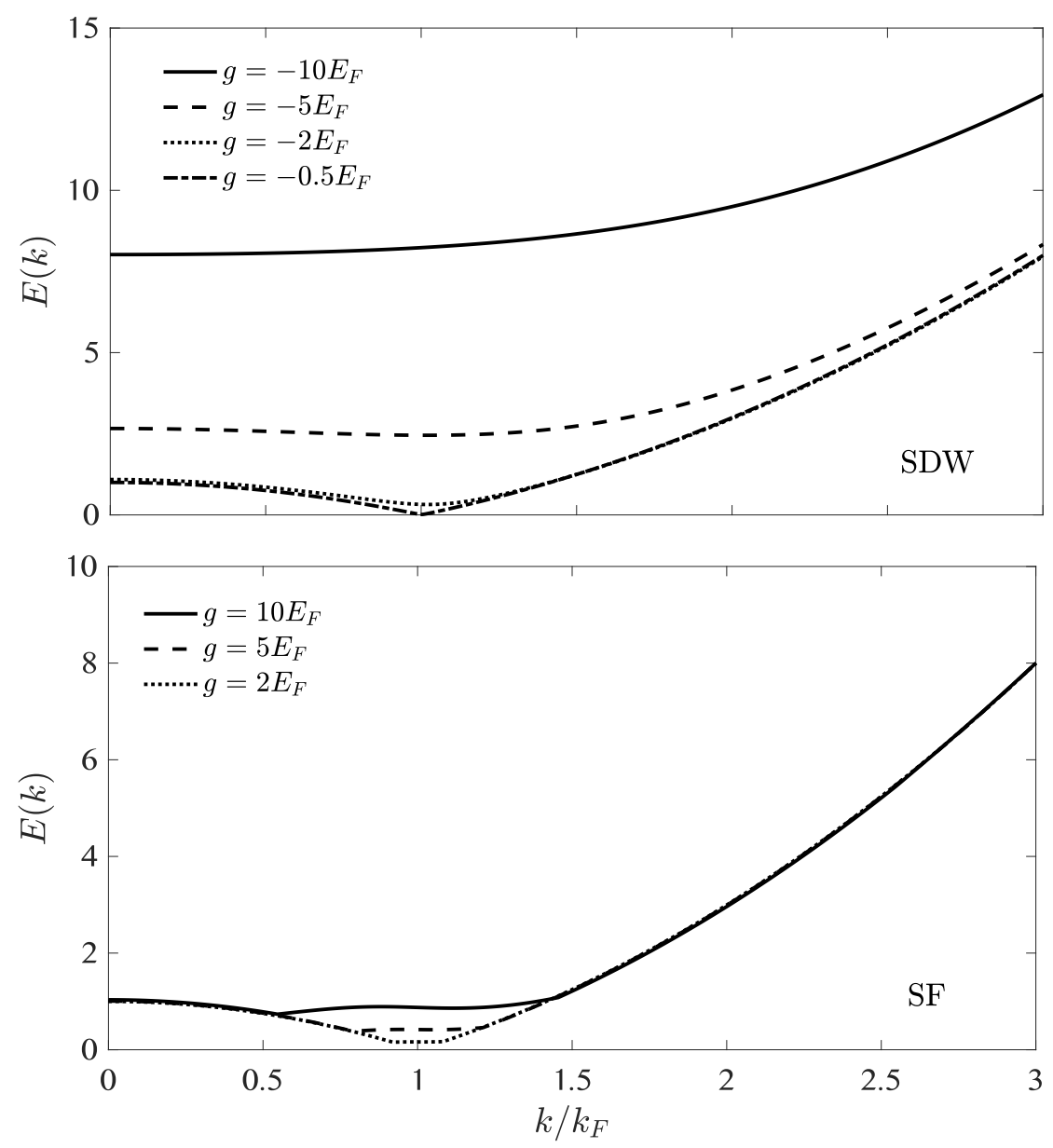

Figure 4. Excitation spectrum $E(k)$ as a function of the momentum, for different values of the interaction strength $g$ as indicated in the legend. Top: SDW region with $g<0$. Bottom: SF region with $g>0$. Energies and momenta are in units of $E_{F}$ and $k_{F}$.

The bottom panel of figure 4 focuses on the behavior of the excitation spectrum in the $\mathrm{SF}$ region $(g>0)$. In this case, the multiple peaks determine the particular structure around $k_{F}$ with the double minima just below and just above $k_{F}$, where the chemical potential compensates the kinetic energy of the atoms and the gaps are visible. Notice that in standard s-wave superfluidity the gap would open up just at the Fermi level. Here instead, two minima manifest, one on the hole side of the spectrum with $k<k_{F}$ and the other on the particle side with $k>k_{F}$. These minima gradually move away from the Fermi level with increasing interactions. In particular, the first minimum shifts towards $k=0$ as the interactions are increased, while the other washes away. This means that more and more particles with smaller momenta are involved in the pairing process, in 
contrast with standard superfluidity where only the particles around the Fermi level are interested.

\subsection{Momentum-distribution function}
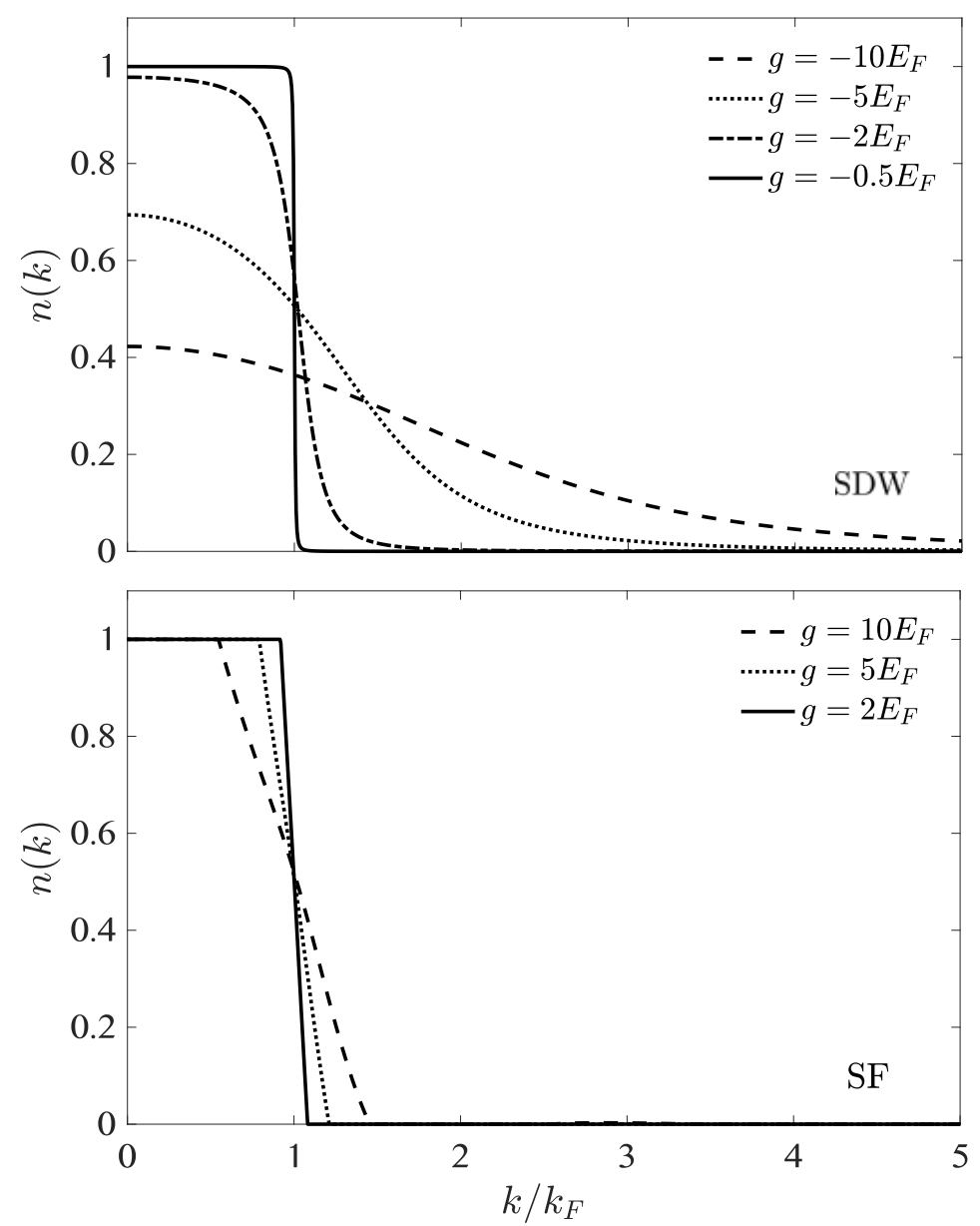

Figure 5. Momentum distribution for different values of the interaction strength $g$ as in the legend. Top: SDW region with $g<0$. Bottom: SF region with $g>0$. Energies and momenta are in units of $E_{F}$ and $k_{F}$.

Additional insight is provided by an analysis of the momentum distribution $n(k)$, which is shown in figure 5 for different values of the interaction strength $g$, and has been obtained within a MF approach. The top panel illustrates the behavior in the SDW region with $g<0$. Drastic differences are found with respect to a conventional Fermi distribution. While the fermionic nature of the fluid remains essentially unchanged for weak interaction strengths $g \approx-1$, at larger values of $g$ the attractive interaction in the particle-hole channel progressively digs inside the Fermi surface and pairs up the particles into composite bosons. This phenomenon is reminiscent of the crossover from a BCS-like to a BEC-like condensation in fermionic systems, originally speculated by Leggett [42] to describe the continuous evolution of the superfluid characteristics with 
increasing the strength of the attractive interaction between fermions in the Cooper pair, and later on demonstrated experimentally in ultracold atoms [1]. However, here the bosonic-like particles are each composed of one particle and one hole. Their possible Bose-Einstein condensation would share analogies with the condensation of excitons, i.e., electron-hole pairs, realized in semiconductor engineered nanostructures.

The bottom panel of figure 5 focuses on the SF region $(g>0)$. The shape of the distribution initially reminds the fermionic nature of the particles. In fact, for low interaction strengths, the momentum distribution shows the discontinuity with size 1 at $k_{F}$, typical of the non-interacting Fermi system. The discontinuity smoothens away after increasing the correlations: interactions drive an increasing number of excitations, moving weight from below to above the Fermi surface. The same trend emerges also in the ED analysis. Here we observed that the shapes of the numerically computed momentum distributions support either a bosonic $(g<0)$ or a fermionic $(g>0)$ character (further details are provided in reference [37]). Bosonization predicts that the momentum distribution has a power-law discontinuity at $k_{F}$ depending on the interaction strength, even at zero temperature [9], but such feature is not well captured by the MF approach.

\subsection{Pair-correlation functions}

We now consider the MF structure of the fluid in real space, as it can be represented by pair-correlation functions of the same spins:

$$
g_{\sigma \sigma}\left(x-x^{\prime}\right)=\frac{1}{n^{2}}\left\langle\hat{\Psi}_{\sigma}^{\dagger}(x) \hat{\Psi}_{\sigma}^{\dagger}\left(x^{\prime}\right) \hat{\Psi}_{\sigma}\left(x^{\prime}\right) \hat{\Psi}_{\sigma}(x)\right\rangle
$$

with $\sigma=\uparrow, \downarrow$, and of opposite spins:

$$
g_{\uparrow \downarrow}\left(x-x^{\prime}\right)=\frac{1}{n^{2}}\left\langle\hat{\Psi}_{\uparrow}^{\dagger}(x) \hat{\Psi}_{\downarrow}^{\dagger}\left(x^{\prime}\right) \hat{\Psi}_{\downarrow}\left(x^{\prime}\right) \hat{\Psi}_{\uparrow}(x)\right\rangle,
$$

with $g_{\uparrow \downarrow}\left(x-x^{\prime}\right)=g_{\downarrow \uparrow}\left(x-x^{\prime}\right)$. In order to better get the picture of the structure in real space, we refer the space distance $r$ to the average particle spacing $r_{0}$, defined by $r_{0}=1 / n=\pi k_{F} / 2$ in terms of the linear density $n$.

Let us start to discuss the behavior of the pair-correlation function for parallel spins. In the SDW region $(g<0$ - see top panel of figure 6$)$, we can recognize a deep well around $r=0$, which is dug in by the Pauli principle and accompanied by Friedel oscillations at $2 r_{0}$; the latter tend to be flattened away for stronger couplings. With respect to the SF side depicted in the bottom panel, on the spin-pairing side the Pauli hole is more effectively replenished by the attractive interactions while the fluid develops its bosonic character discussed so far and the average size of the correlation hole is even shorter than $r_{0}$. At the same time, the amplitude of the Friedel oscillations damps much more rapidly in the strong coupling regime, where the correlation energy mostly resides in the channel of the opposite spins.

Looking at the SF region $(g>0)$, - see bottom panel of figure 6 - we observe that, even if the pair-distribution function does not explicitly depend on the superfluid 


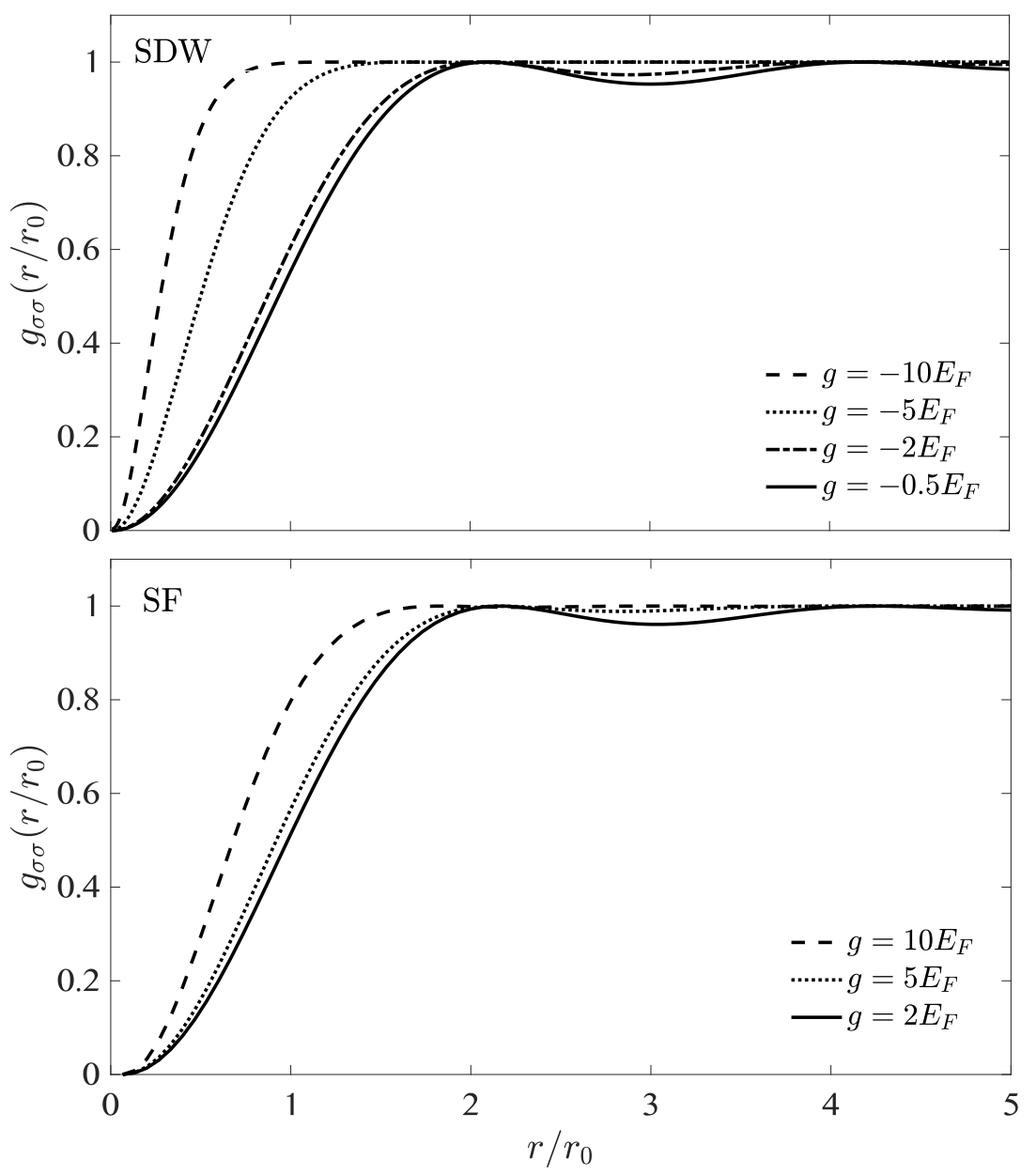

Figure 6. Spin-parallel pair-distribution function $g_{\uparrow \uparrow}\left(r / r_{0}\right)=g_{\downarrow \downarrow}\left(r / r_{0}\right)$ at different interaction strengths $g$, as indicated in the legend. Top: SDW region with $g<0$. Bottom: SF region with $g>0$.

order parameter, effects of interactions are indirectly included in the occupation number, and manifest themselves as a change in the width of the distribution, which is more evident at strong coupling. At large distances the distribution tends to unity as expected for homogeneous systems. The weakly oscillating behavior is instead a manifestation of Friedel oscillations, ultimately connected to the existence of a Fermi surface and of singular behavior in correspondence of $k_{F}$. As in the SDW region discussed above, for larger values of $g$ and when the interaction energy prevails over the kinetic one, the system tends to localize and oscillations are damped. Similar behaviors have been predicted by simulational methods in the investigation of the 1D Hubbard model [43], where actually a damping of Friedel oscillations at $2 k_{F}$ is also accompanied by a building up of oscillations at $4 k_{F}$ while the system undergoes a Wigner crystallization, that is a freezing of the quantum fluid into a crystal. Appropriate inclusion of density fluctuations, here neglected, and a full numerical simulation in 1D would be needed to understand to which extent such a prediction might apply to the present system as well. 

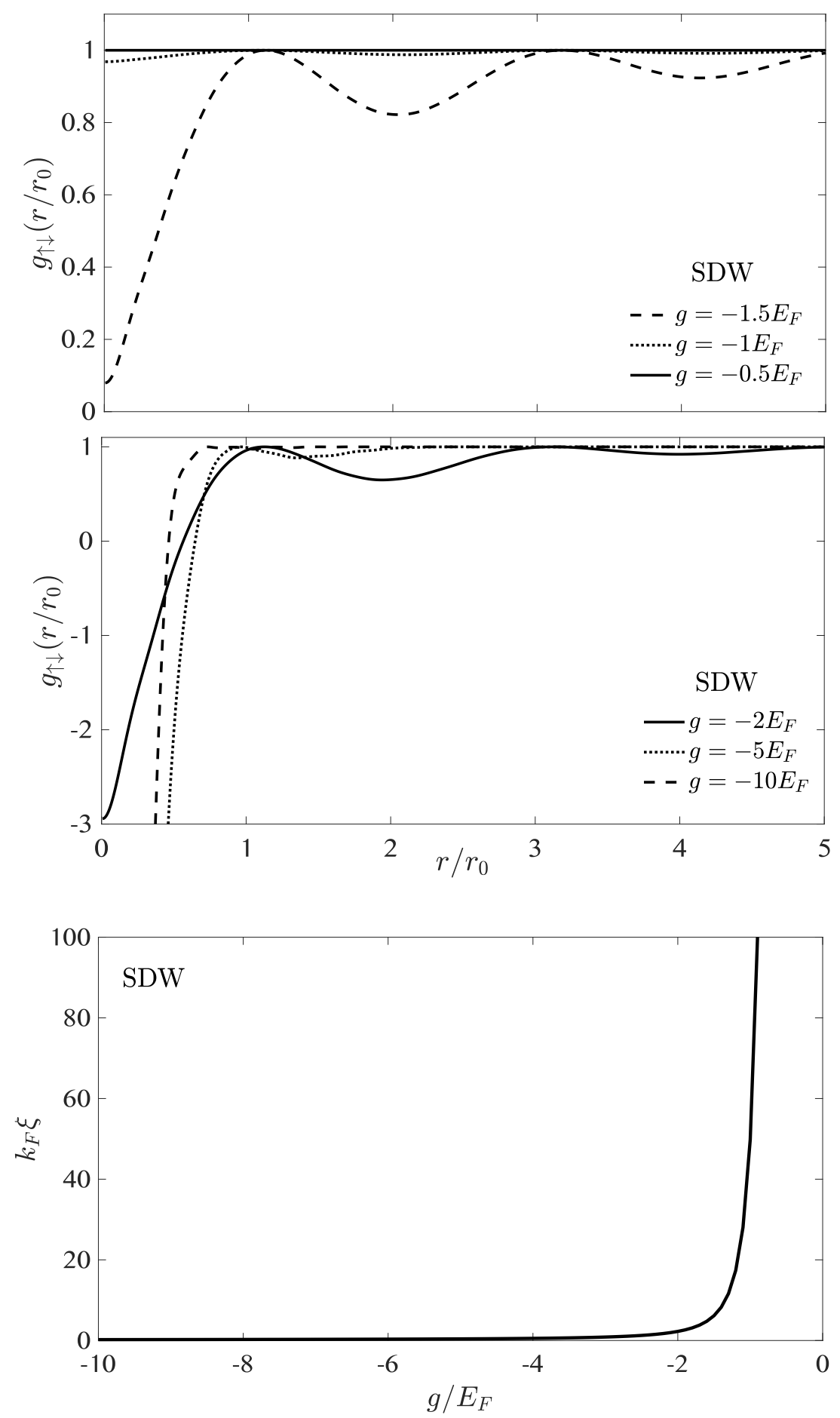

Figure 7. The spin-antiparallel pair-distribution function $g_{\uparrow \downarrow}\left(r / r_{0}\right)=g_{\downarrow \uparrow}\left(r / r_{0}\right)$ in the SDW regime, top panel for weak interaction and middle panel for strong interactions. The correlation length is shown in the SDW regime in the bottom panel. 

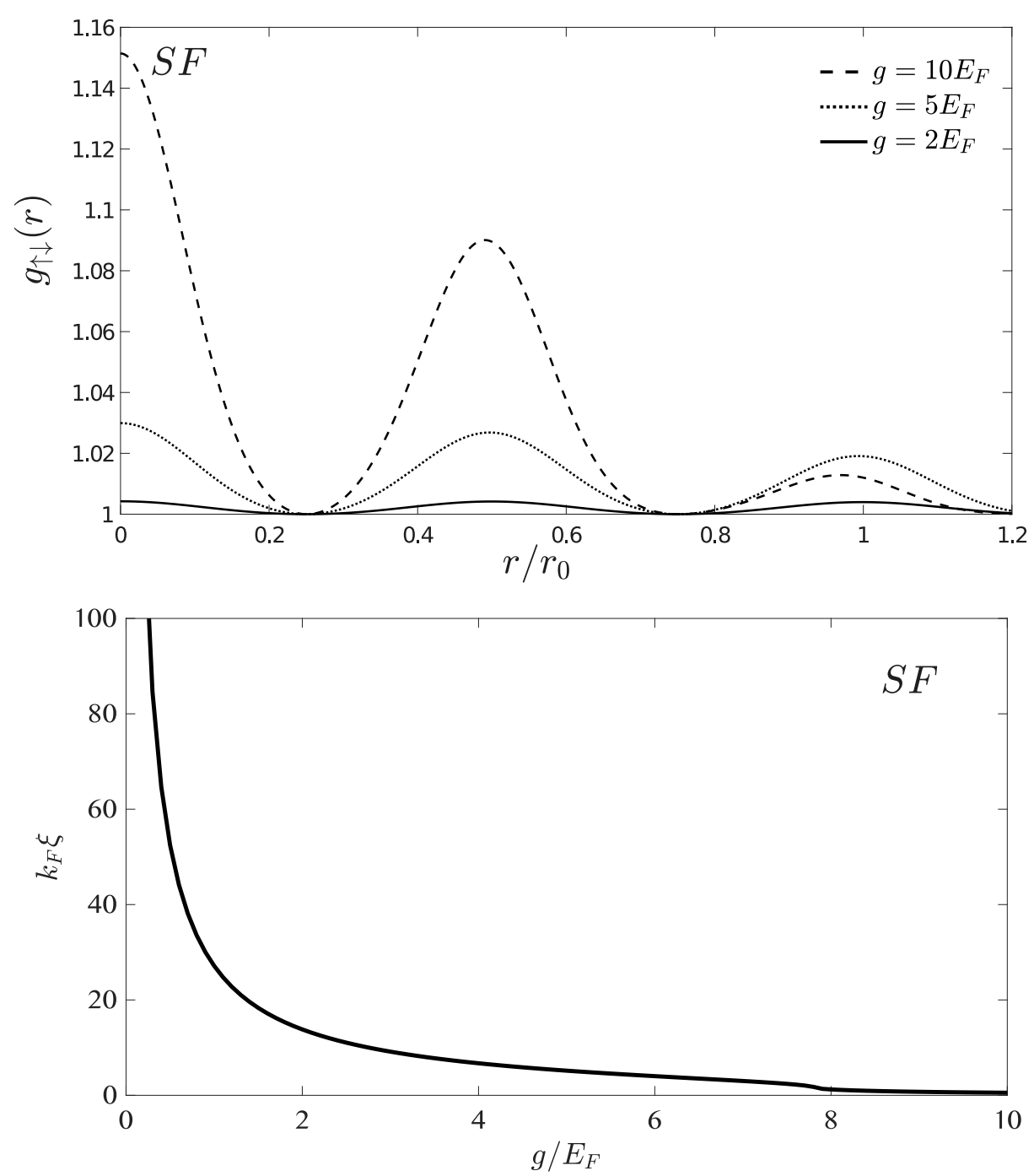

Figure 8. The spin-antiparallel pair-distribution function $g_{\uparrow \downarrow}\left(r / r_{0}\right)=g_{\downarrow \uparrow}\left(r / r_{0}\right)$ in the SF regime.

Let us now come to the description of the opposite-spin pair-correlation function $g_{\uparrow \downarrow}$, which is depicted in figure 7 and figure 8. In the SDW region $(g<0$, figure 7$)$, it is characterized by periodic peaks at odd multiples of the average interparticle distance $r_{0}$, thus shifted by $r_{0}$ with respect to its superfluid counterpart in figure 8 . This suggests that spin ordering occurs, as expected, in antiferromagnetic configuration. In both cases, the periodicity can be traced back to the long-range nature of the interactions. However, here the rapid amplitude decay of the peaks even for weak couplings (top panel in figure 7) suggests that the particle-hole channel to be favoured with respect the superfluid one. This is not surprising, since the effective Hamiltonian privileges direct coupling between spin rather than particle variables. At stronger coupling (middle panel in figure 7) the well around $r=0$ becomes even deeper and oscillations are enhanced, hinting a stronger antiferromagnetic character of the fluid which self-organizes. However 
we note that, at strong interactions, the MF theory overestimates correlations, this can be seen in the fact that for strong $g$ the pair-correlation function becomes negative.

The increasing bosonic character appears evident from the analysis of the paircorrelation length

$$
k_{F} \xi=\left\{\int d x x^{2}\left[g_{\uparrow \downarrow}(x)-1\right]\right\}^{1 / 2}\left\{\int d x\left[g_{\uparrow \downarrow}(x)-1\right]\right\}^{-1 / 2} .
$$

The pair-correlation length is a quantitative measure of the average size of the pairs, in fact the square root of the second moment of the pair-correlation function $g_{\sigma \sigma^{\prime}}(x)$. The latter, in turn, represents indeed the probability of finding a second particle with spin $\sigma$ at distance $x$ from a given first particle, given the positions of all the other particles and the interactions among them. As shown in the bottom panel of figure 7 , strong attractive interactions tie up the particle-hole pairs in real space, resulting into tiny pair-correlation lengths, while weak interactions favor delocalized periodic spin pairing with large values of $k_{F} \xi$. The pair-distribution function $g_{\uparrow \downarrow}$ in the SF region, with $g>0$, is shown in the top panel of figure 8. Qualitative deep differences can be found with respect to the standard BCS case, where the pair-distribution function consists of a structure around $r=0$, whose height increases with the interaction strength, its width being the correlation length $\xi_{\mathrm{SF}}$ measuring the average size of the Cooper pair. Here, the pair-distribution function is periodic with period $2 r_{0}$, signaling - in accordance with the results on the SF gap function - the emergence of nonlocal superfluidity with periodic character similar to what is named Fulde-Ferrel-Larkin-Ovchinnikov (FFLO)
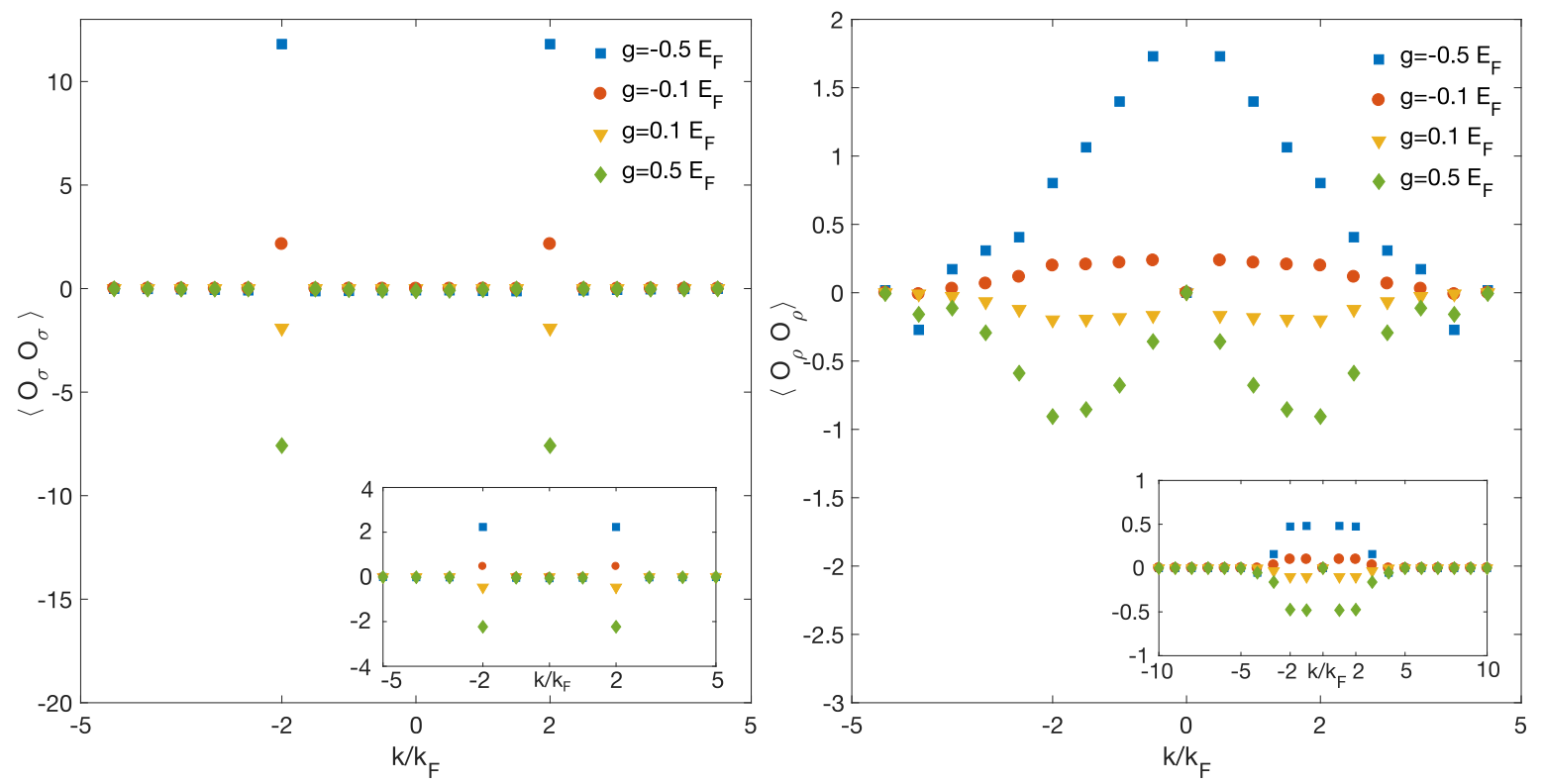

Figure 9. Correlation functions in $k$-space for different $g$ values, as obtained with ED (see reference [37]). Spin-spin correlation $\left\langle\hat{O}_{\sigma} \hat{O}_{\sigma}\right\rangle$ (left panel) and density-density correlation $\left\langle\hat{O}_{\rho} \hat{O}_{\rho}\right\rangle$ (right panel). Data in the main panels are for $N=8$, while the same quantities with $N=4$ are shown in the insets. Adapted from [37]. 
phase $[44,45]$. Similar inhomogeneous superconducting states were also studied via bosonization for fermions with attractive interaction in a quasi 1D configuration [45]. Here, the oscillations are mainly due to the characteristic long-range nature of the interactions in our system. The height of the replicas at multiples of twice $r_{0}$ appears only weakly decreasing for weak coupling strengths $g<1$. We see that the heights significantly drop down for stronger interactions, while the oscillations in the same-spin pair-correlation function damps down. Overall, such a behavior suggests that SF ordering has a delocalized nature, which is progressively lost while the coupling strength increases. This can be viewed also in terms of the pair-correlation length $k_{F} \xi$, which we find to symmetrically parallel the behavior in the SDW region, that is diverging while the interaction parameter vanishes, and fast dropping down at larger $g$ values, see bottom panel in figure 8.

Finally, figure 9 displays the $k$-space correlation functions for density $\left\langle\hat{O}_{\rho} \hat{O}_{\rho}\right\rangle$ and spin $\left\langle\hat{O}_{\sigma} \hat{O}_{\sigma}\right\rangle$ fluctuations. Such results come from an ED analysis, and have been detailed in reference [37]. In order to make the presentation self contained, here we recall their main features and complement them with an extended discussion. In the density-density correlation function, we notice the peak developing at $k=0$ for $g<0$, and not for $g>0$; peaks at $\pm 2 k_{F}$ emerge only for $g>0$ signaling the setting of ADW processes. In the spin-spin correlation function, we see the expected appearance of the peaks at $\pm 2 k_{F}$, driven by SDW and ADW processes. The SDW behavior is confirmed by the bosonization analysis that confirms the opening of a gap in the spin sector for $g>0$ and above a certain strength of $g$, based on a renormalization group analysis. In the case of ADW ordering, the combination of ED and bosonization analysis suggests that ADW is originated by higher-energy processes, in fact not captured at the lowest order of renormalization group analysis. This conclusion supports the choice of leaving ADW on a side in the simplest MF approach.

\section{Conclusions}

In conclusion, we have studied the fluid structure characterizing the quantum phases of a 1D spinful atomic Fermi gas, where spin and motional degrees of freedom are both relevant, and interacting via an infinitely long-ranged effective interaction with variable strength and sign. Following our previous work [37], based on a combined mean-field, exact diagonalization and bosonization analysis, we have discussed in detail the structure of the quantum fluid in order provide additional insight on the phase diagram, including spin- and atomic-density wave and by superfluid-like orderings, ultimately favored by nesting of the Fermi points.

In particular, we have systematically analyzed the $k$-space structure of gaps, excitation spectra, and momentum distribution, along with the same- and opposite-spin pair-correlation functions expressing the structure of the fluid in real space. We have used the mean-field analysis as a track for the discussion, due to its qualitative reliability in the presence of (infinitely) long-range interactions, ex-post confirmed in the comparison 
with exact diagonalization and bosonization results. Whenever useful, the mean-field outcomes have been complemented with those obtained from exact diagonalization and from bosonization analysis. In particular, we have discussed the extent to which the exact-diagonalization and bosonization analysis qualitatively support the mean-field results.

The clues in our hands suggest that on the $g>0$ side one might have a BCS-like superfluid state, which evolves from an unconventional FFLO-like periodic character at weak coupling strengths, towards a more conventional state for larger interactions. They also suggest that the $g<0$ side might be characterized, for weak coupling strengths, by a spatially ordered state similar to the one for $g>0$, but developing in the particle-hole channel and in the form of an antiferromagnetic arrangement. In fact, looking again at the pair-correlation function, we find periodic structures at $r_{0}$ in the ones for opposite spins and $2 r_{0}$ in the ones for parallel spins. This can be interpreted as correlations between particles characterized by an ordered structure, as if correlated fermions with opposite spins arrange at relative distance of $r_{0}$ (and multiples) while correlated fermions with parallel spins are created at distance $2 r_{0}$ (and multiples). With increasing strength of the interactions, the antiferromagnetic spin ordering appears to be lost, evolving into a structure where particles with opposite spins are tied to form composite bosonic pairs with diamagnetic character, which Bose-Einstein condense. In any event, an evident asymmetry characterizes the order parameter sizes on the two sides of the interaction strength, since the photon scattering processes are directly coupled to spin fluctuations and only indirectly lead to a superfluid pairing.

Our predictions can be in principle realized in quantum atomic Fermi gases in optical cavities. However, a realistic implementation necessarily requires the inclusion of the intrinsic out-of-equilibrium physics, to be tackled with methods different than those exploited in the present work. The proper inclusion of optical-cavity dissipation mechanisms is needed in order to establish the amount and conditions, which the physics here discussed persists to.

\section{Acknowledgments}

One of us (MLC) acknowledges funding from the MIT-UNIPI program and from INFN under the MAGIA-Advanced project.

\section{References}

[1] S. Giorgini, L. P. Pitaevskii, and S. Stringari. Theory of ultracold Fermi gases. Rev. Mod. Phys. 80, 1215 (2008).

[2] F. Dalfovo, S. Giorgini, L. P. Pitaevskii, and S. Stringari. Theory of Bose-Einstein condensation in trapped gases. Rev. Mod. Phys. 71, 463 (1999).

[3] I. Bloch, J. Dalibard, and W. Zwerger. Many-body physics with ultracold gases. Rev. Mod. Phys. 80, 885 (2008). 
[4] H. Chen, Y. Ren, Y. Qiu, Wei Bao, R. H. Liu, G. Wu, T. Wu, Y. L. Xie, X. F. Wang, Q. Huang, and X. H. Chen. Coexistence of the spin-density wave and superconductivity in $\mathrm{Ba}_{1-x} \mathrm{~K}_{x} \mathrm{Fe}_{2} \mathrm{As}_{2}$. Europhys. Lett. 85, 17006 (2009).

[5] K. Kudo, Y. Nishikubo, and M. Nohara. Coexistence of superconductivity and charge density wave in $\mathrm{SrPt}_{2} \mathrm{As}_{2}$. J. Phys. Soc. Jpn. 79, 123710 (2010).

[6] T. Desta, G. Kahsay, and P. Singh. Coexistence of superconductivity and spin density wave (sdw) in ferropnictide $\mathrm{Ba}_{1-x} \mathrm{~K}_{x} \mathrm{Fe}_{2} \mathrm{As}_{2}$. J. Supercond. Nov. Magn. 29, 1433 (2016).

[7] G. Iadonisi, J. R. Schrieffer, and M. L. Chiofalo. Models and Phenomenology for Conventional and High-Temperature Superconductivity. Società Italiana di Fisica IOS Press, Proceedings of the international school Enrico Fermi Course CXXXVI, 1998.

[8] D. Manske. Theory of Unconventional Superconductors: Cooper-Pairing mediated by spin excitations. Springer, 2002.

[9] T. Giamarchi. Quantum Physics in One Dimension. Oxford Univ. Press, 2003.

[10] H. Tanji-Suzuki, I. D. Leroux, M. H. Schleier-Smith, M. Cetina, A. T. Grier, J. Simon, and V. Vuletić. Interaction between atomic ensembles and optical resonators: Classical description. Adv. At. Mol. Opt. Phys. 60, 201 (2011).

[11] H. Ritsch, P. Domokos, F. Brennecke, and T. Esslinger. Cold atoms in cavitygenerated dynamical optical potential. Rev. Mod. Phys. 85, 553 (2013).

[12] F. Mivehvar, F. Piazza, and H. Ritsch. Disorder-driven density and spin self-ordering of a Bose-Einstein condensate in a cavity. Phys. Rev. Lett. 119, 063602 (2017).

[13] F. Mivehvar, H. Ritsch, and F. Piazza. Superradiant topological Peierls insulator inside an optical cavity. Phys. Rev. Lett. 118, 073602 (2017).

[14] S. Ostermann, H.-W. Lau, H. Ritsch, and F. Mivehvar. Cavity-induced emergent topological spin textures in a Bose-Einstein condensate. New J. Phys. 21, 013029 (2018).

[15] F. Mivehvar, H. Ritsch, and F. Piazza. Cavity-quantum-electrodynamical toolbox for quantum magnetism. Phys. Rev. Lett. 122, 113603 (2019).

[16] E. Colella, S. Ostermann, W. Niedenzu, F. Mivehvar, and H. Ritsch. Antiferromagnetic self-ordering of a Fermi gas in a ring cavity. New J. Phys. 21, 043019 (2019).

[17] S. F. Caballero-Benitez and I. B. Mekhov. Bond order via light-induced synthetic many-body interactions of ultracold atoms in optical lattices. New J. Phys. 18, 113010 (2016).

[18] V. D. Vaidya, Y. Guo, R. M. Kroeze, K. E. Ballantine, A. J. Kollár, J. Keeling, and B. L. Lev. Tunable-range, photon-mediated atomic interactions in multimode cavity qed. Phys. Rev. X 8, 011002 (2018). 
[19] A. T. Black, H. W. Chan, and V. Vuletić. Observation of collective friction forces due to spatial self-organization of atoms: From Rayleigh to Bragg scattering. Phys. Rev. Lett. 91, 203001 (2003).

[20] J. K. Asbóth, P. Domokos, H. Ritsch, and A. Vukics. Self-organization of atoms in a cavity field: Threshold, bistability, and scaling laws. Phys. Rev. A 72, 053417 (2005).

[21] D. Nagy, G. Szirmai, and P. Domokos. Self-organization of a Bose-Einstein condensate in an optical cavity. Eur. Phys. J. D 48, 127 (2008).

[22] K. Baumann, C. Guerlin, F. Brennecke, and T. Esslinger. Dicke quantum phase transition with a superfluid gas in an optical cavity. Nature 464, 1301 (2010).

[23] K. Baumann, R. Mottl, F. Brennecke, and T. Esslinger. Exploring symmetry breaking at the Dicke quantum phase transition. Phys. Rev. Lett. 107, 140402 (2011).

[24] J. Keeling, M. J. Bhaseen, and B. D. Simons. Fermionic superradiance in a transversely pumped optical cavity. Phys. Rev. Lett. 112, 143002 (2014).

[25] F. Piazza and P. Strack. Umklapp superradiance from a collisionless quantum degenerate fermi gas. Phys. Rev. Lett. 112, 143003 (2014).

[26] M. Xu, S. B. Jäger, S. Schütz, J. Cooper, G. Morigi, and M. J. Holland. Supercooling of atoms in an optical resonator. Phys. Rev. Lett. 116, 153002 (2016).

[27] A. Sheikhan, F. Brennecke, and C. Kollath. Cavity-induced generation of nontrivial topological states in a two-dimensional Fermi gas. Phys. Rev. A 94, 061603 (2016).

[28] A. Sheikhan and C. Kollath. Cavity-induced superconducting and $4 k_{-} f$ chargedensity-wave states. Phys. Rev. A 99, 053611 (2019).

[29] C.-M. Halati, A. Sheikhan, and C. Kollath. Cavity-induced artificial gauge field in a Bose-Hubbard ladder. Phys. Rev. A 96, 063621 (2017).

[30] J. Léonard, A. Morales, P. Zupancic, T. Esslinger, and T. Donner. Supersolid formation in a quantum gas breaking a continuous translational symmetry. Nature 543, 87 (2017).

[31] J. Léonard, A. Morales, P. Zupancic, T. Donner, and T. Esslinger. Monitoring and manipulating Higgs and goldstone modes in a supersolid quantum gas. Science $\mathbf{3 5 8}$, 1415 (2017).

[32] M. Landini, N. Dogra, K. Kroeger, L. Hruby, T. Donner, and T. Esslinger. Formation of a spin texture in a quantum gas coupled to a cavity. Phys. Rev. Lett. 120, 223602 (2018).

[33] E. J Davis, G. Bentsen, L. Homeier, T. Li, and M. H. Schleier-Smith. Photonmediated spin-exchange dynamics of spin-1 atoms. Phys. Rev. Lett. 122, 010405 (2019).

[34] D. Yu, J.-S. Pan, X.-J. Liu, W. Zhang, and W. Yi. Topological superradiant state in Fermi gases with cavity induced spin-orbit coupling. Front. Phys. 13, 136701 (2018). 
[35] F. Mivehvar and D. L. Feder. Synthetic spin-orbit interactions and magnetic fields in ring-cavity qed. Phys. Rev. A 89, 013803 (2014).

[36] X. Guo, Z. Ren, G. Guo, and J. Peng. Ultracold fermi gas in a single-mode cavity: Cavity-mediated interaction and bcs-bec evolution. Phys. Rev. A 86, 053605 (2012).

[37] E. Colella, R. Citro, M. Barsanti, D. Rossini, and M.-L. Chiofalo. Quantum phases of spinful Fermi gases in optical cavities. Phys. Rev. B 97, 134502 (2018).

[38] J. Fan, X. Zhou, W. Zheng, W. Yi, G. Chen, and S. Jia, Magnetic order in a Fermi gas induced by cavity-field fluctuations Phys. Rev. A 98, 043613 (2018).

[39] A. Camacho-Guardian, R. Paredes, and S. F. C.-Benítez. Quantum simulation of competing orders with fermions in quantum optical lattices. Phys. Rev. A 96, 051602(R) (2017).

[40] S. F. Caballero-Benitez and I. B. Mekhov. Quantum Optical Lattices for Emergent Many-Body Phases of Ultracold Atoms. Phys. Rev. Lett. 115, 243604 (2015).

[41] G. Mazzucchi, S. F. Caballero-Benitez, and I. B. Mekhov. Quantum measurementinduced antiferromagnetic order and density modulations in ultracold Fermi gases in optical lattices. Sci. Rep. 6, 31196 (2016).

[42] A. Leggett. Diatomic molecules and Cooper pairs. Proceedings of the XVI Karpacz Winter School of Theoretical Physics, February 19 - March 3, 1979, Karpacz, Poland, Springer, 1980.

[43] S. A. Soffing, M. Bortz, I. Schneider, A. Struck, M. Fleischhauer, and S. Eggert. Wigner crystal versus Friedel oscillations in the one-dimensional Hubbard model. Phys. Rev. B 79, 195114 (2009).

[44] H. Shimahara. Theory of the Fulde-Ferrell-Larkin-Ovchinnikov State and Application to Quasi-Low-dimensional Organic Superconductors in: The Physics of Organic Superconductors and Conductors. Springer Series in Materials Science Volume 110, 2008.

[45] K. Yang. Inhomogeneous superconducting state in quasi-one-dimensional systems. Phys. Rev. B 63, 140511(R) (2001).

\section{Appendix A. Adiabatic elimination of cavity fields}

Consider $N$ three-level atoms interacting with the electromagnetic field of a single-mode linear cavity of frequency $\omega_{c}$. The existence of a privileged direction given by the cavity axis, suggests to restrict the analysis to a one-dimensional geometry. As schematized in figure A1, the atomic transition is transversely pumped by a classical field with frequency $\omega_{p}$ driving the $|s\rangle \leftrightarrow|e\rangle$ transition. The cavity field induces a transition between a second ground state and the same excited state, $|g\rangle \leftrightarrow|e\rangle$. In terms of the atomic and 
photonic creation and annihilation operators, the Hamiltonian can be represented as:

$$
\begin{aligned}
\hat{H}(t)= & \int d x\left[\frac{\hbar \omega_{g s}}{2}\left(\hat{\Psi}_{s}^{\dagger}(x) \hat{\Psi}_{s}(x)-\hat{\Psi}_{g}^{\dagger}(x) \hat{\Psi}_{g}(x)\right)+\hbar \omega_{e} \hat{\Psi}_{e}^{\dagger}(x) \hat{\Psi}_{e}(x)\right]+\hbar \omega_{c} \hat{a}^{\dagger} \hat{a} \\
& +\hbar \Omega \int d x\left(e^{i \omega_{p} t} \hat{\Psi}_{s}^{\dagger}(x) \hat{\Psi}_{e}(x)+e^{-i \omega_{p} t} \hat{\Psi}_{e}^{\dagger}(x) \hat{\Psi}_{s}(x)\right) \\
& +\hbar g_{0} \int d x \cos \left(k_{L} x\right)\left(\hat{\Psi}_{e}^{\dagger}(x) \hat{\Psi}_{g}(x) \hat{a}+\hat{a}^{\dagger} \hat{\Psi}_{g}^{\dagger}(x) \hat{\Psi}_{e}(x)\right) .
\end{aligned}
$$

Here, the first line represents bare-atom and bare-photon Hamiltonian, where the motional degrees of freedom are temporarily frozen. The energy difference between the two ground states $|s\rangle$ and $|g\rangle$ is $\omega_{g s}$, and $\omega_{e}$ is the energy of the excited level $|e\rangle$. The first term in the second line is the classical pump with strength $\hbar \Omega$, while the last term represents the coherent interaction between the atom and cavity mode with strength $\hbar g_{0}$, where the mode function in one dimension is $\cos \left(k_{L} x\right)$. After applying the unitary transformation

$$
\hat{U}(t)=e^{i \omega_{p}\left(\int d x\left[\hat{\Psi}_{s}^{\dagger} \hat{\Psi}_{s}-\hat{\Psi}_{g}^{\dagger} \hat{\Psi}_{g}+\hat{\Psi}_{e}^{\dagger} \hat{\Psi}_{e}\right]+\hat{a}^{\dagger} \hat{a}\right) t}
$$

the time independent Hamiltonian, $\hat{H} \rightarrow \hat{U}^{\dagger}(t) \hat{H}(t) \hat{U}(t)-i \hbar \hat{U}^{\dagger}(t) \partial \hat{U}(t) / \partial t$, in the frame rotating with the pump field $\omega_{p}$, is

$$
\begin{aligned}
\hat{H} & =\int d x\left[-\frac{\hbar \Delta_{g s}}{2}\left(\hat{\Psi}_{s}^{\dagger}(x) \hat{\Psi}_{s}(x)-\hat{\Psi}_{g}^{\dagger}(x) \hat{\Psi}_{g}(x)\right)-\hbar \Delta_{e} \hat{\Psi}_{e}^{\dagger}(x) \hat{\Psi}_{e}(x)\right]-\hbar \Delta_{c} \hat{a}^{\dagger} \hat{a} \\
& +\hbar \Omega \int d x\left(\hat{\Psi}_{s}^{\dagger}(x) \hat{\Psi}_{e}(x)+\hat{\Psi}_{e}^{\dagger}(x) \hat{\Psi}_{s}(x)\right) \\
& +\hbar g_{0} \int d x \cos \left(k_{L} x\right)\left(\hat{\Psi}_{e}^{\dagger}(x) \hat{\Psi}_{g}(x) \hat{a}+\hat{a}^{\dagger} \hat{\Psi}_{g}^{\dagger}(x) \hat{\Psi}_{e}(x)\right) .
\end{aligned}
$$

Here, all energies are measured with reference to the cavity pump frequency: $\Delta_{c}=$ $\omega_{p}-\omega_{c}, \Delta_{g s}=\omega_{p}-\omega_{g s}$ and $\Delta_{e}=\omega_{p}-\omega_{e}$. In the large detuning regime for $\Delta_{e}$, the $\hat{\Psi}_{e}(x)$ state is only virtually populated and can be adiabatically eliminated. Thus, we

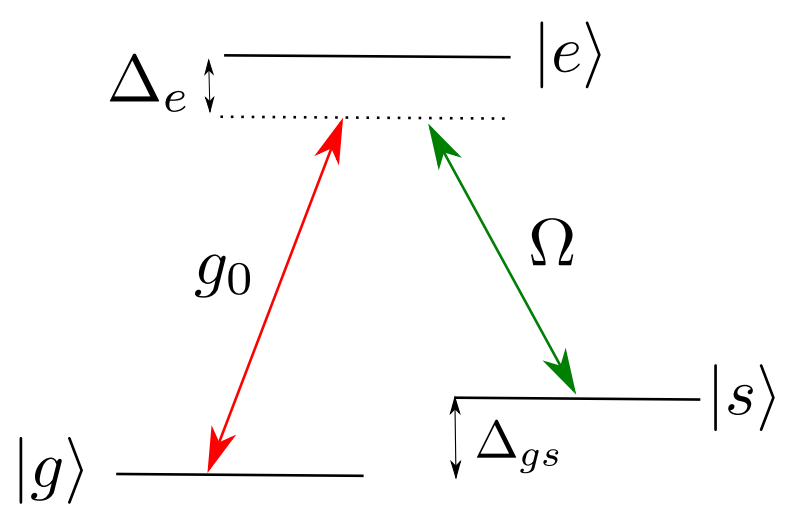

Figure A1. Pumping configuration leading to the Hamiltonian (1) in the main text. The $|g\rangle \leftrightarrow|e\rangle$ transition is driven by cavity photons and the $|s\rangle \leftrightarrow|e\rangle$ by an external transversal pump beam. 
can set to zero the fast time evolution of the excited state $|e\rangle$ :

$$
i \hbar \frac{\partial \hat{\Psi}_{e}(x)}{\partial t}=-\hbar \Delta_{e} \hat{\Psi}_{e}(x)+\hbar \Omega \hat{\Psi}_{s}(x)+\hbar g_{0} \cos \left(k_{L} x\right) \hat{a} \hat{\Psi}_{g}(x)=0 .
$$

Inserting the stationary value

$$
\hat{\Psi}_{e}(x)=\frac{\Omega}{\Delta_{e}} \hat{\Psi}_{s}(x)+\frac{g_{0}}{\Delta_{e}} \cos \left(k_{L} x\right) \hat{\Psi}_{g}(x) \hat{a}
$$

into equation (A.3), the following effective two-level Hamiltonian is obtained:

$$
\begin{aligned}
\hat{H}_{3 L}= & \int d x\left[\hat{\Psi}_{s}^{\dagger}(x)\left(\delta_{s}-\frac{\hbar \Delta_{g s}}{2}\right) \hat{\Psi}_{s}(x)+\hat{\Psi}_{g}^{\dagger}(x)\left(\frac{\hbar \Delta_{g s}}{2}+V_{0}(x) \hat{a}^{\dagger} \hat{a}\right) \hat{\Psi}_{g}(x)\right] \\
& -\hbar \Delta_{c} \hat{a}^{\dagger} \hat{a}+\hbar g_{\mathrm{eff}} \int d x \cos \left(k_{L} x\right)\left(\hat{\Psi}_{s}^{\dagger}(x) \hat{\Psi}_{g}(x) \hat{a}+\hat{a}^{\dagger} \hat{\Psi}_{g}^{\dagger}(x) \hat{\Psi}_{s}(x)\right) .
\end{aligned}
$$

Notice that the two-photon transition induces a classical shift $\delta_{s}=\hbar \Omega^{2} / \Delta_{e}$ on the effective ground-state level, $\hat{\Psi}_{s}$. The other ground state, $\hat{\Psi}_{e}$, experiences a periodic potential dependent on the number of photons within the cavity $V_{0}(x)=$ $\hbar g_{0}^{2} \cos ^{2}\left(k_{L} x\right) / \Delta_{e}$. Coupling between the two levels is mediated via an effective twophoton Rabi frequency, which now depends on the pump strength, that is $g_{\text {eff }}=g_{0} \Omega / \Delta_{e}$.

When the cavity photons evolve over a faster time-scale compared to the atomic dynamics, the photonic operators can be slaved to the atomic ones. This happens either in the bad cavity regime or for large cavity detunings $\Delta_{c}$. In this case, the photonic operator in equation (A.6) can be substituted by their stationary values obtained by setting to zero the Heisenberg equation of motion for these operators

$$
\dot{\hat{a}}=-\left(\Delta_{c}+i \frac{\kappa}{2}\right) \hat{a}+g_{\mathrm{eff}} \int d x \cos \left(k_{L} x\right) \hat{\Psi}_{g}^{\dagger}(x) \hat{\Psi}_{s}(x)=0 .
$$

Here, $\kappa$ is loss rate related to cavity dissipation. Note that the coupling to the ground state density, $V_{0}=\hbar g_{0}^{2} / \Delta_{e}$, depends only on the dipole moment of the transition $|g\rangle \leftrightarrow|e\rangle$ and is neglected in equation (A.7). In fact, this term is much smaller than the two-photon Rabi frequency $g_{\text {eff }}=g_{0} \Omega / \Delta_{e}$, which can become very large for high intensity of the pumping laser, $\Omega$. In order to obtain the Hamiltonian (1) in the main text, we first plug equation (A.7) into (A.6) and include the kinetic energy of the atoms. Then, we neglect the state-dependent light shift $\delta_{s}$, which can be compensated by any additional classical light-shift. We also neglect the optical lattice $V_{0} \cos ^{2}\left(k_{L} x\right) \hat{a}^{\dagger} \hat{a}$, which acts only on the ground state $|g\rangle$, for the same reasons as above. Collecting together all these approximations, the Hamiltonian (1) in the main text is obtained.

\section{Appendix B. Fully coupled gap and number equations in mean-field theory}

After having tested the solution of the problem in separate regimes as shown in the main text, the fully coupled problem can be more easily addressed, where both order 
parameters are assumed to be nonzero. We report here from reference [37] the main steps in the derivation.

First, an explicit ansatz for the superfluid gap function can be introduced, guided by the fact that the superfluid gap equation (6) has solution only when the interaction parameter $g$ is positive, since the right-hand-side is a positive-definite quantity. The opposite occurs for the spin-pairing gap equation (9), where $g<0$ is required instead. Therefore, at the present mean-field level, a first guess for the superfluid and spin order parameters is the following: given the value of $g$, set $\Delta_{\mathrm{SF}}=0$ and $\Delta_{S P}$ at the value found from the solution of (9)-(11) if $g<0$, and set $\Delta_{\mathrm{SP}}=0$ and $\Delta_{\mathrm{SF}}$ at the value found from the solution of (6)-(8) if $g>0$. For the determination of $\Delta(k)$, we therefore assume that the function that expresses its dependence on $k$ can be easily parametrized. It is assumed that $\Delta(k)$ can be expressed as a product between a function having $4 k_{F}$ periodicity and an envelope function consisting of a plateau for $|k| \leq 4 k_{F}$, exponentially dropping down in size at $|k|>4 k_{F}$. The first factor can be extended to the whole $k$ space using the values of $\Delta$ in the interval $\left[-2 k_{F}, 2 k_{F}\right]$ and the condition $\Delta(k)=\Delta\left(k+4 k_{F}\right)$. The resulting function can be inserted in the complete set of equations of the problem and finally the following set of coupled equations for the three unknown quantities $N, \Delta$ and $S$ is obtained:

$$
\begin{aligned}
N & =2 \sum_{k}\left\{\frac{1}{2}-\frac{1}{4}\left[1+\frac{\varepsilon_{-}(k)}{E_{s}(k)}\right] \frac{\epsilon_{k}}{E_{+}(k)} \tanh \left(\frac{\beta E_{+}(k)}{2}\right)\right. \\
& \left.-\frac{1}{4}\left[1-\frac{\varepsilon_{-}(k)}{E_{s}(k)}\right] \frac{\epsilon_{k}}{E_{-}(k)} \tanh \left(\frac{\beta E_{-}(k)}{2}\right)\right\}, \\
\Delta(k) & =\frac{g}{4} \sum_{k} V_{k k^{\prime}}\left\{\left[\Delta\left(k^{\prime}\right)+\left(E_{s}\left(k^{\prime}\right)+\frac{\left(\Delta\left(k^{\prime}\right)\right)^{2}-\left(\Delta\left(k^{\prime}-Q\right)\right)^{2}}{E_{s}\left(k^{\prime}\right)}\right)\right] \frac{\tanh \left(\beta E_{+}(k) / 2\right)}{E_{+}\left(k^{\prime}\right)}\right. \\
& \left.+\left[\Delta\left(k^{\prime}\right)-\left(E_{s}\left(k^{\prime}\right)+\frac{\left(\Delta\left(k^{\prime}\right)\right)^{2}-\left(\Delta\left(k^{\prime}-Q\right)\right)^{2}}{E_{s}\left(k^{\prime}\right)}\right)\right] \frac{\tanh \left(\beta E_{-}(k) / 2\right)}{E_{-}\left(k^{\prime}\right)}\right\}, \quad \text { (B.1b) } \\
S(q) & =-\frac{g}{4} \sum_{k}\left\{\frac{S(q)}{E_{+}(k)} \tanh \left(\frac{\beta E_{+}(k)}{2}\right)+\frac{S(q)}{E_{+}(k)} \tanh \left(\frac{\beta E_{+}(k)}{2}\right)\right\},
\end{aligned}
$$

where we have defined $\varepsilon_{ \pm}(k) \equiv \Delta(k) \pm \Delta(k+Q), E_{s}(k) \equiv \sqrt{\left[\varepsilon_{-}(k)\right]^{2}+\Delta_{\text {SDW }}^{2}}$, and the single-particle energies $E_{ \pm}(k) \equiv \sqrt{\epsilon_{k}^{2}+\left[\varepsilon_{+}(k) \pm E_{s}(k)\right]^{2}}$. 


\section{Appendix C. Bosonization method: Renormalization-Group equations}

In this Appendix we derive the equations for the $R G$ equations closely following reference [9]. We start from the action

$$
\begin{aligned}
S & =\sum_{l=\rho, \sigma} \frac{1}{2 \pi} \iint d \tau d x\left\{u_{l} K_{l}\left[\left(\partial_{\tau} \hat{\theta}_{l}\right)^{2}+\left(\partial_{x} \hat{\theta}_{l}\right)^{2}\right]+\frac{u_{l}}{K_{l}}\left[\left(\partial_{\tau} \hat{\phi}_{l}\right)^{2}+\left(\partial_{x} \hat{\phi}_{l}\right)^{2}\right\}\right. \\
& +\frac{g}{4 \pi^{2} \alpha^{2}} \iiint d \tau d x d x^{\prime} e^{-i\left(\hat{\theta}_{\sigma}(x, \tau)-\hat{\theta}_{\sigma}\left(x^{\prime}, \tau\right)\right)} \cos \left(\sqrt{2}\left(\hat{\phi}_{\rho}(x, \tau)-\hat{\phi}_{\rho}\left(x^{\prime}, \tau\right)\right) .\right.
\end{aligned}
$$

If one imposes a sharp momentum cutoff $\Lambda$ and varies the cut-off between $\Lambda$ and $\Lambda^{\prime}$, the field can be decoupled in slow modes, whose Fourier transform is limited to $|q|<\Lambda^{\prime}$, and fast modes where $\Lambda^{\prime}<|q|<\Lambda$.

After defining

$$
\hat{\phi}_{l}(r)=\hat{\phi}_{l}^{>}(r)+\hat{\phi}_{l}^{<}(r), \quad \hat{\theta}_{l}(r)=\hat{\theta}_{l}^{>}(r)+\hat{\theta}_{l}^{<}(r),
$$

where $r=(x, \tau)$, the quadratic part of the action can be decoupled, as well in $S_{0}=S_{0}^{<}+S_{0}^{<}$. If one now takes the partition function

$$
Z=\int \mathcal{D} \theta_{\rho} \mathcal{D} \phi_{\rho} \mathcal{D} \theta_{\sigma} \mathcal{D} \phi_{\sigma} e^{-S\left[\hat{\theta}_{\rho}, \hat{\phi}_{\rho}, \hat{\theta}_{\sigma}, \hat{\phi}_{\sigma}\right]}
$$

the exponential can be expanded in series of the interaction Hamiltonian up to the first non-zero order

$$
\begin{aligned}
Z & =\int \mathcal{D} \theta_{\rho} \mathcal{D} \phi_{\rho} \mathcal{D} \theta_{\sigma} \mathcal{D} \phi_{\sigma} e^{-S_{0}}\left[1-\frac{g}{4 \pi^{2} \alpha^{2}} \iiint d \tau d x d x^{\prime} e^{-i\left(\hat{\theta}_{\sigma}(x, \tau)-\hat{\theta}_{\sigma}\left(x^{\prime}, \tau\right)\right)} \times\right. \\
& \times \cos \left(\sqrt{2}\left(\hat{\phi}_{\rho}(x, \tau)-\hat{\phi}_{\rho}\left(x^{\prime}, \tau\right)\right)\right] .
\end{aligned}
$$

After averaging over the fast modes, we are left with an effective Hamiltonian for the slow modes only, i.e.

$$
\begin{aligned}
Z & =\int \mathcal{D} \theta_{\rho} \mathcal{D} \phi_{\rho} \mathcal{D} \theta_{\sigma} \mathcal{D} \phi_{\sigma} e^{-S_{0}^{<}}\left[1-\frac{g}{4 \pi^{2} \alpha^{2}} \iiint d \tau d x d x^{\prime} e^{-i\left(\hat{\theta}_{\sigma}^{<}(x, \tau)-\hat{\theta}_{\sigma}^{<}\left(x^{\prime}, \tau\right)\right)} \times\right. \\
& \times \cos \left(\sqrt{2}\left(\hat{\phi}_{\rho}^{<}(x, \tau)-\hat{\phi}_{\rho}^{<}\left(x^{\prime}, \tau\right)\right) e^{-\left\langle\left(\hat{\phi}_{\rho}^{>}(x, \tau)-\hat{\phi}_{\rho}^{>}\left(x^{\prime}, \tau\right)\right)^{2}\right\rangle} e^{-\left\langle\left(\hat{\theta}_{\sigma}^{>}(x, \tau)-\hat{\theta}_{\sigma}^{>}\left(x^{\prime}, \tau\right)\right)^{2}\right\rangle}\right] .
\end{aligned}
$$

We now define $\Delta \hat{\theta}_{\sigma}=\sqrt{2}\left(\hat{\theta}_{\sigma}^{<}(x, \tau)-\hat{\theta}_{\sigma}^{<}\left(x^{\prime}, \tau\right)\right)$ and $\Delta \hat{\phi}_{\rho}=\sqrt{2}\left(\hat{\phi}_{\rho}^{<}(x, \tau)-\hat{\phi}_{\rho}^{<}\left(x^{\prime}, \tau\right)\right)$. The correction to the effective action is thus

$$
\left.S_{0}-\frac{g}{4 \pi^{2} \alpha^{2}} \iiint d \tau d x d x^{\prime} e^{-i \Delta \hat{\theta}_{\sigma}\left(x, x^{\prime}\right)} \cos \left(\Delta \hat{\phi}_{\rho}\left(x, x^{\prime}\right)\right) e^{-\left\langle\left[\Delta \hat{\phi}_{\rho}\left(x, x^{\prime}\right)\right]^{2}\right\rangle} e^{-\left\langle\left[\Delta \hat{\theta}_{\sigma}\left(x, x^{\prime}\right)\right]^{2}\right\rangle}\right],
$$

Here one has

$$
e^{-\left\langle\left[\Delta \hat{\phi}_{\rho}\left(x, x^{\prime}\right)\right]^{2}\right\rangle} e^{-\left\langle\left[\Delta \hat{\theta}_{\sigma}\left(x, x^{\prime}\right)\right]^{2}\right\rangle}=e^{-\left(K_{\rho}+\frac{1}{K_{\sigma}}\right) \int_{\Lambda^{\prime}<\mathbf{q}<\Lambda}\left[1-\cos \left(q\left(x-x^{\prime}\right)\right] \frac{2 \pi u}{\omega^{2}+u^{2} q^{2}}\right.},
$$


where $\omega$ is a small quantity going to zero. The second term can be cast in the form

$$
\begin{aligned}
& -\frac{g}{4 \pi^{2} \alpha^{2}} \iiint d \tau d x d x^{\prime} e^{-i \Delta \hat{\theta}_{\sigma}\left(x, x^{\prime}\right)} \cos \left(\Delta \hat{\phi}_{\rho}\left(x, x^{\prime}\right)\right) e^{-\left(K_{\rho}+\frac{1}{K_{\sigma}}\right) \int_{\Lambda^{\prime}<\mathbf{q}<\Lambda} \frac{2 \pi u}{\omega^{2}+u^{2} q^{2}}} \\
& -\frac{g}{4 \pi^{2} \alpha^{2}} \iiint d \tau d x d x^{\prime} e^{-i \Delta \hat{\theta}_{\sigma}\left(x, x^{\prime}\right)} \cos \left(\Delta \hat{\phi}_{\rho}\left(x, x^{\prime}\right)\right) e^{-\left(K_{\rho}+\frac{1}{K_{\sigma}}\right) \int_{\Lambda^{\prime}<\mathbf{q}<\Lambda} \frac{2 \pi u}{\omega^{2}+u^{2} q^{2}} \times} \\
& \times\left[-1+e^{-\left(K_{\rho}+\frac{1}{K_{\sigma}}\right) \int_{\Lambda^{\prime}<\mathbf{q}<\Lambda} \cos \left(q\left(x-x^{\prime}\right)\right) \frac{2 \pi u}{\omega^{2}+u^{2} q^{2}}}\right]
\end{aligned}
$$

The first line in (C.7) represents the renormalization of the coupling constant. In fact, it has the same shape of the bare interaction Hamiltonian for the slow modes with an effective coupling constant. To recover the original action, one should rescale time and space, and bring back the original cutoff so that $d x=\Lambda / \Lambda^{\prime} d x^{\prime}$ and $d \tau=\Lambda / \Lambda^{\prime} / d \tau^{\prime}$. One thus obtains:

$$
g\left(\Lambda^{\prime}\right)=\left(\frac{\Lambda}{\Lambda^{\prime}}\right)^{3} g(\Lambda) e^{-\left(K_{\rho}+\frac{1}{K_{\sigma}}\right) \int_{\Lambda^{\prime}<\mathbf{q}<\Lambda} \frac{2 \pi u}{\omega^{2}+u^{2} q^{2}}} .
$$

With the parametrization $\Lambda(l)=\Lambda_{0} e^{-l}$, we get the most general RG equation

$$
\frac{d g(l)}{d l}=g(l)\left(3-K_{\rho}-\frac{1}{K_{\sigma}}\right) .
$$

The second and third line in (C.7) lead to the renormalization of $K_{\rho}$ and $K_{\sigma}$. In order to derive the corresponding relations, we introduce the center-of-mass $R=\left(x+x^{\prime}\right) / 2$ and relative-motion $r=x-x^{\prime}$ coordinates, and expand both cosine and exponential terms in powers of $r \sim 0$ :

$$
e^{-i \Delta \hat{\theta}_{\sigma}\left(x, x^{\prime}\right)} \cos \left(\Delta \hat{\phi}_{\rho}\left(x, x^{\prime}\right)\right) \simeq 1-\frac{r^{2}}{2}\left(\nabla \hat{\theta}_{\sigma}\right)^{2}-\frac{r^{2}}{2}\left(\nabla \hat{\phi}_{\rho}\right)^{2} .
$$

Consistently, the exponential in the third line is expanded in the cosine argument up to first order. The correction to the action becomes

$$
\delta I=g\left(K_{\rho}+\frac{1}{K_{\sigma}}\right) \iint d \tau d R\left[\left(\partial_{R} \hat{\theta}_{\sigma}\right)^{2}+\left(\partial_{R} \hat{\phi}_{\rho}\right)^{2}\right] \int d r r^{2} e^{-\left(K_{\rho}+\frac{1}{K_{\sigma}}\right) F_{\Lambda}(r)} J_{0}(\Lambda r),
$$

with

$$
F_{\Lambda}(r)=\int_{|q|<\Lambda}\left[1-\cos \left(q\left(x-x^{\prime}\right)\right)\right] \frac{2 \pi u}{\omega^{2}+u^{2} q^{2}}=\int_{0}^{\Lambda} \frac{d q}{q}\left(1-J_{0}(q r)\right),
$$

in the limit $\omega \rightarrow 0$. We are now in the position to write down the RG equations for both $K_{\sigma}$ and $K_{\rho}$, after considering that the velocity $u_{l}$ is not renormalized as a consequence of Lorentz invariance. Therefore we get

$$
\begin{aligned}
& \frac{d\left(u_{\rho} K_{\rho}\right)}{d l}=0 \\
& \frac{d\left(u_{\rho} / K_{\rho}\right)}{d l}=2 \pi G\left(K_{\rho}+\frac{1}{K_{\sigma}}\right) f\left(K_{\rho}, K_{\sigma}\right), \\
& \frac{d\left(u_{\sigma} K_{\sigma}\right)}{d l}=2 \pi G\left(K_{\rho}+\frac{1}{K_{\sigma}}\right) f\left(K_{\rho}, K_{\sigma}\right), \\
& \frac{d\left(u_{\sigma} / K_{\sigma}\right)}{d l}=0
\end{aligned}
$$


so that $u_{\sigma} / K_{\sigma}=$ cost $=c_{\sigma}$ and $u_{\rho} K_{\rho}=$ cost $=c_{\rho}$ and finally the complete set of RG equations

$$
\begin{aligned}
\frac{d K_{\rho}}{d l} & =-\frac{\pi G K_{\rho}^{3}}{c_{\rho}}\left(K_{\rho}+\frac{1}{K_{\sigma}}\right) f\left(K_{\rho}, K_{\sigma}\right), \\
\frac{d K_{\sigma}}{d l} & =\frac{\pi G}{c_{\sigma} K_{\sigma}}\left(K_{\rho}+\frac{1}{K_{\sigma}}\right) f\left(K_{\rho}, K_{\sigma}\right), \\
\frac{d G(l)}{d l} & =G(l)\left(3-K_{\rho}-\frac{1}{K_{\sigma}}\right),
\end{aligned}
$$

with

$$
f\left(K_{\rho}, K_{\sigma}\right)=\int d r r^{2} e^{-\left(K_{\rho}+\frac{1}{K_{\sigma}}\right) F_{\Lambda}(r)} J_{0}(\Lambda r) .
$$

From these equations one could get the RG flow lines and determine the values of $K_{\rho}$ and $K_{\sigma}$ at which the coupling $G$ becomes relevant and the flow must be stopped. 